\title{
Effect of orthodontic forces on cytokine and receptor levels in gingival crevicular fluid: a systematic review
}

\author{
Priyanka Kapoor ${ }^{1}$, Om Prakash Kharbanda ${ }^{2 *}$, Nitika Monga ${ }^{2}$, Ragini Miglani ${ }^{1}$ and Sunil Kapila ${ }^{3}$
}

\begin{abstract}
This systematic review aimed to generate evidence on role of potent markers of inflammation [cytokines, chemokines, their associated receptors and antagonists] following the application of orthodontic forces. Subsequent to registration with PROSPERO, literature search followed a predetermined search strategy to key databases along with hand search (HS). Seventy-seven articles from PubMed (P), 637 from Scopus (S), 51 from Embase (E), and 3 from hand search (HS) were identified. A total of 39 articles were shortlisted that met strict inclusion and exclusion criteria and quality assessment. Each study was evaluated for participant characteristics, study design, oral hygiene regimen, and gingival crevicular fluid (GCF) handling. Among these studies, biomarkers in the order of frequency were interleukin (IL)-1 $\beta(N=21)$, tumor necrosis factor (TNF)- $a(N=10), I L-8, I L-6(N=8)$, receptor activator of nuclear factor kappa-B ligand (RANKL) $(N=7)$, monocyte chemoattractant protein (MCP)-1 ( $N=3), I L-2(N=4), I L-4, I L-10$, RANTES $(N=2), I L-1, I L-5$, IL-1a, IP-10, osteopontin (OPN) (N=1) and receptors and their antagonists in the order of osteoprotegerin (OPG) $(N=8), I L-1 R A(N=5)$, and RANK $(N=1)$. Results revealed an immediate release of inflammatory bone-resorptive mediators, IL-1 $\beta$ and TNF-a, where IL-1 $\beta$ increased as early as $1 \mathrm{~min}$ to $1 \mathrm{~h}$ reaching peak at $24 \mathrm{~h}$ while TNF-a increased at $1 \mathrm{~h}$ or 1 day. This was accompanied by a fall in bone-protective mediator (OPG) levels at $1 \mathrm{~h}$ and $24 \mathrm{~h}$ after orthodontic force application. Continuous forces were accompanied by a decrease in mediator levels after attaining peak levels (most commonly at $24 \mathrm{~h}$ ) while repeated activations in interrupted force upregulated their secretion. Significant correlations of IL-1 $\beta$ levels with pain intensity, rate of orthodontic tooth movement (OTM) and of activity index (Al) (IL-1B/LL-1RA) with velocity of tooth movement and growth status of individuals have also been deduced. A greater Al and RANKL/OPG ratio was seen in juveniles as compared to adults or non-growers that were associated with faster rate of OTM in juveniles. None of the studies addressed the effect of estrous cycle in female subjects. Lack of homogeneity in several parameters calls for a better controlled research on the biology of OTM.
\end{abstract}

Keywords: GCF; Cytokines; Chemokines; Receptors; IL-13; RANK/RANKL; OPG

\section{Review}

\section{Background}

Orthodontic tooth movement (OTM) is considered an epiphenomenon of the gene expression of the periodontal ligament (PDL) and neighboring cells resulting from a series of orchestrated cellular and molecular events in alveolar bone and periodontal tissue initiated by the application of orthodontic force [1]. A chemical cascade

\footnotetext{
* Correspondence: opk15@hotmail.com

${ }^{2}$ Division of Orthodontics and Dentofacial Deformities, Centre for Dental Education and Research, All India Institute of Medical Sciences, New Delhi 110029, India

Full list of author information is available at the end of the article
}

that mediates the transmission of signals from extracellular matrix leading to genetic modulation is interceded by the release of mediators in paracrine environment. These signals are responsible for a change in the cytoskeletal structure, leading to alteration of nuclear protein matrix and eventually gene activation or suppression $[2,3]$. These events initiate the process of bone remodeling, leading to effective tooth movement. The biochemical mediators released sequentially at multiple stages during orchestration of tooth movement can be detected in gingival crevicular fluid (GCF). GCF is a unique biological exudate that has been found as a convenient medium to study these mediators with reasonable sensitivity. GCF can be 
collected noninvasively [4] with specifically designed filter paper or a micropipette ( 1 to $10 \mu \mathrm{l}$ ) or through magnetic beads placed in gingival crevice. GCF once collected may be cryopreserved or directly sent for chemical analysis. GCF can also be collected repeatedly at various stages of orthodontic treatment and therefore provides useful insight to biological events over the entire duration of observation.

Clinically demonstrable success of OTM is associated with expression of numerous regulatory molecules, of which cytokines have been most widely documented. Cytokines are low-molecular weight proteins $(\mathrm{mw}<$ $25 \mathrm{kDa}$ ) released in autocrine or paracrine environment in response to local signals like application of stress [5] and are involved in normal physiological bone turnover and remodeling [6-8]. Cytokine biology as a retort to forces applied for OTM is difficult to comprehend due to sheer number and complexity of these factors exhibiting redundancy as well as pleiotropy [9]. Although cytokines have been extensively evaluated in GCF as quantitative biochemical indicators of inflammatory periodontal status [10], there has been an increasing interest on understanding their contributions as mediators of OTM owing to their role in bone and tissue remodeling. Among these cytokines, interleukins (ILs) (IL-1 $\alpha$, IL-1 $\beta$, IL-1RA, IL-8, IL-2, IL-6, and IL-15), tumor necrosis factors (TNFs), interferons (IFNs), growth factors (GFs), and colonystimulating factors (CSFs) have been extensively studied in relation to OTM.

The secretion of these mediators in the local environment by cells activated on application of orthodontic force varies according to the force levels and functional state of available target cells. The rate of OTM depends on recruitment of mature osteoclasts and precursors, osteoclast differentiation and number of functional osteoclasts at the bone-PDL interface, where bone resorption is considered a rate-limiting step [11]. The earliest identified marker of bone resorption is IL-1 $\beta$, closely followed by prostaglandin E2 (PGE2), nitric oxide, IL-6, and other inflammatory cytokines [12]. TNF is also believed to have synergistic effects with IL-1 [13]. Osteoclast differentiation and activation is mediated by the binding of receptor activator of nuclear factor kappa-B to its ligand expressed by osteoblasts and PDL cells (RANK and RANKL, respectively) [11,14-16]. This interaction and osteoclast activity is prevented by a decoy receptor for RANKL called osteoprotegerin (OPG) [17]. Thus, the relative balance between RANK-RANKL and OPG may be critical to the magnitude and rate of OTM.

The first experimental evidence supporting role of cytokines in OTM was documented in periodontal tissues of cat canine teeth where IL- $1 \alpha$ and IL- $1 \beta$ were identified after the application of a tipping force [18]. One of the earliest noninvasive studies on IL-1 $\beta$ in GCF was done by Grieve et al. [19] where significant elevations from baseline in IL-1 $\beta$ and PGE levels supported initial release of proinflammatory mediators on application of orthodontic forces, followed by a decrease in 7 days (d). Since then, numerous studies in humans have focused on alterations in IL-1 $\beta$ levels in GCF in an attempt to understand the underlying inflammatory process during OTM. The studies have now diversified to include other interleukins like IL-1 $\alpha$, IL-2, IL-6, IL-8, and receptor antagonist IL-1RA. More recently, the presence of other mediators including TNF- $\alpha$, TGF- $\beta$, leptin, RANK/RANKL, and OPG have also been explored in OTM.

This systematic review aims to determine effect of orthodontic forces on levels of markers of inflammation namely cytokines, chemokines, receptors and their antagonists, which have been widely documented in GCF. The present study looks into literature to generate evidence on the role of these mediators in relation to the force levels, applied mechanics, age, sex and pain intensity during orthodontic treatment. This systematic review provides insights into possible biomarkers for tooth movement and their potential contributions to modulating orthodontic bone turnover that could prove useful in designing future approaches to modulating OTM.

\section{Material and methods}

This review was registered in PROSPERO on 3 June, 2014 (registration number CRD42014009302). The systematic review was conducted strictly adhering to guidelines suggested by PROSPERO. A search strategy was finalized utilizing MESH terms, Boolean terminology, and free text terms (Additional file 1: Annexure 1). This search strategy was applied to key databases PubMed, Scopus, and Embase in April 2014 by two reviewers PK and NM which followed a cross check by third reviewer OPK. Apart from that, hand search of journals was performed for article retrieval. Segregation of articles to be considered for review was based on stringent inclusion and exclusion criteria. Seventy-seven articles in PubMed, 637 in Scopus, 51 in Embase, and 3 from hand searching were retrieved (Figure 1). Duplicates were removed before final screening of articles for inclusion in the review. The titles and abstracts of these manuscripts were studied, considering exclusion and inclusion criteria specific to each database (Figure 1). The relevant articles were obtained. These were PubMed - 41, Scopus - 17, Embase - 2 and hand searched - 3 . Further, a few articles had to be excluded for non-availability of full text. These were PubMed $(\mathrm{N}=4)$, Scopus $(\mathrm{N}=15)$, and Embase $(\mathrm{N}=1)$. Further, four articles had to be excluded since full text revealed no mention of orthodontic force $(\mathrm{N}=1)$ or study was not performed in crevicular fluid $(\mathrm{N}=3)$. A consensus has arrived among reviewers for final inclusion of 39 
articles comprising of 33 articles from PubMed, 2 from Scopus, 1 from Embase, and 3 from hand search (Figure 1).

The inclusion and exclusion criterion were as under.

\section{Inclusion Criteria:}

Participants/population -Human studies, age groups (if specified), male to female ratio (if specified), controls (either internal where baseline levels are taken as control or external where contralateral or antagonistic tooth is taken as control), sample size $>5$ (refers to sample size not number of teeth studied).

Intervention(s), exposure(s)-Studies on cytokines [including interleukins (ILs), tumor necrosis factor (TNF) and growth factors (GFs), colony-stimulating factors (CSFs), interferons (IFNs)], chemokines, receptors and their antagonists (RANK, RANKL, OPG, OPN) with specified orthodontic mechanics, proper oral hygiene control, no use of antibiotic/ anti-inflammatory drugs before or during

\begin{tabular}{|c|c|}
\hline IDENTIFICATION & \\
\hline $\begin{array}{l}\text { Citations Screened from Electronic } \\
\text { Databases } \\
\text { Pubmed-77 } \\
\text { Scopus-637 } \\
\text { Embase-51 }\end{array}$ & $\begin{array}{l}\text { Records after Duplicates Removed } \\
\text { Pubmed-77 } \\
\text { Scopus-549 } \\
\text { Embase-13 } \\
\text { Handsearched-3 }\end{array}$ \\
\hline $\begin{array}{l}\text { Citation Screened from } \\
\text { Handsearching-3 }\end{array}$ & \\
\hline $\begin{array}{l}\text { Excluded Citations } \\
\text { Pubmed } \\
\begin{array}{l}\text { - } \\
\text { - }\end{array} \text { Not related to mediators under study-17 } \\
\text { - In vitro-3 } \\
\text { - Not related to ortho TM-4 } \\
\text { - Not in GCF-2 } \\
\text { - Studies related to periodontitis-0 } \\
\text { - Review studies-6 }\end{array}$ & $\begin{array}{l}\text { Relevant Articles Identified } \\
\text { Pubmed-41 } \\
\text { Scopus-17 } \\
\text { Embase-2 } \\
\text { Handsearched-3 }\end{array}$ \\
\hline 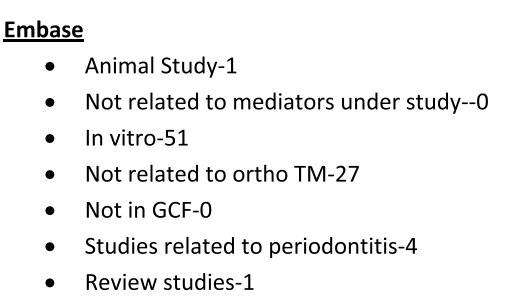 & $\begin{array}{l}\text { Exclusion based on non retrieval in full } \\
\text { text for detailed evaluation- } \\
\text { Pubmed-4 } \\
\text { Scopus-15 } \\
\text { Embase-1 }\end{array}$ \\
\hline $\begin{array}{l}\text { - Studies involving drug effects in ortho TM-0 } \\
\text { Scopus } \\
\text { - Animal Study-56 } \\
\text { - Not related to mediators under study-86 }\end{array}$ & $\begin{array}{l}\text { Further exclusions } \\
\text { Ortho force/mechanics not mentioned-1 } \\
\text { In sulcus but not in crevicular fluid-3 }\end{array}$ \\
\hline $\begin{array}{l}\text { - } \text { In vitro-124 } \\
\text { - Not related to ortho TM-26 } \\
\text { - } \text { Studies related to periodontitis-67 } \\
\text { - } \text { Review studies-54 } \\
\text { - Studies involving drug effects in OTM-40 } \\
\text { - Unrelated-43 }\end{array}$ & $\begin{array}{l}\text { Citation chosen for the systemic review } \\
\text { Pubmed-33 } \\
\text { Scopus-2 } \\
\text { Embase-1 } \\
\text { Handsearched-3 }\end{array}$ \\
\hline
\end{tabular}

Figure 1 Flowchart depicting the retrieval of studies for review process. 
orthodontic force application, GCF sample collection via periopaper or micropipette placed in sulcus.

\section{Exclusion criteria}

Participants/population- In vitro studies, animal studies, sample size $<5$, no control. Intervention(s), exposure(s)- Studies on mediators other than cytokines or chemokines or receptors, cytokine or chemokine or receptor measurement in periodontal tissue and not GCF, cytokine or chemokine or receptor levels consequent to periodontal inflammation and not orthodontic force application, cytokine levels measured in peri-implant fluid.

This is a followed data extraction by two reviewers (PK and NM). The data was recorded in a tabular form based on the following criteria:

1. Participant characteristics (Table 1): number of study subjects (not the number of teeth), teeth considered for study (if specified), sites (if mentioned), age of study subjects (either range or mean age \pm standard deviation (SD)), sex, controls, and studied mediators. Apart from these, following inclusion criteria were also considered (if mentioned) no history of drug intake, no bone loss, no gingival inflammation, and pocket depth $<2 \mathrm{~mm}$.

2. Study characteristics (Table 2): these were nature of applied force, force magnitudes, force reactivations (if studied), total study duration, observation intervals, and type of tooth movement.

3. Oral hygiene regimen and assessment of gingival health (Table 3): recommendation and frequency of mouthwash intake, oral prophylaxis schedule, use of indices for gingival and periodontal assessment, and their frequency.

4. GCF characteristics (Table 4): time, room temperature and humidity during GCF collection, site, method of collection of GCF, storage and handling characteristics, and technique of mediator analysis.

Quality assessment of the articles included in the review was done based on a Quality Assessment Instrument (QAI) modified and developed from relevant articles in literature [20,21] given in Additional file 2: Annexure 2.

Thirty stringent criteria for evaluation of quality included relevant study design $(\mathrm{N}=19)$, study measurements $(\mathrm{N}=3)$, statistical analysis $(\mathrm{N}=5)$, study results, and conclusions $(\mathrm{N}=3)$. (Additional file 2: Annexure 2). For objective assessment of quality determination, a scoring system was incorporated where scores of 1 to 10 were considered minimal, 10 to 20 were considered moderate, and 20 to 30 were considered highly sensitive. QAI revealed 26 moderately sensitive and 13 highly sensitive studies. None of the studies fell in the score of 1 to 10 .

The results were compiled after grouping of observations from similar studies to arrive at conclusions with relevant clinical implications.

\section{Results}

Thirty-nine shortlisted studies [19,22-59] were scrutinized for inclusion and exclusion criteria. Two studies that evaluated mediators both in peri-implant crevicular fluid (PMICF) as well as GCF were included [51,54] but one study that evaluated the levels of mediators directly in sulcus and not GCF was excluded [60].

The studies were categorized based on participant characteristics (Table 1), study characteristics (Table 2), oral hygiene regimen and assessment of gingival health (Table 3), and GCF characteristics (Table 4). All studies displayed control, either internal/external. The levels of biomarkers assessed at baseline level ( 0 day) were taken as control in the former while in the later, contralateral or antagonistic teeth were taken as control. GCF sampling was done with either using periopaper or micropipette that were placed in the gingival sulcus. An overview of the results obtained has been summarized in Table 5.

\section{Sample characteristics \\ Sample size}

Of the 39 studies, the sample size varied, smallest being 7 subjects [40] to a maximum of 84 subjects [38]. The studies were categorized in four groups, with sample size up to $10(\mathrm{~N}=15), 11$ to $20(\mathrm{~N}=16), 21$ to $30(\mathrm{~N}=5)$, and 31 and above $(\mathrm{N}=3)$. Average sample size taken was 10 subjects $(\mathrm{N}=10)$.

\section{Sex predilection}

Information on sex of the subjects was mentioned in $\mathrm{N}$ $=36$ studies. One study $(\mathrm{N}=1)$ included only male subjects. Equal numbers of male and female subjects constituted the sample in ten studies $(\mathrm{N}=10)$.

\section{Age distribution}

Age was expressed as either range or as mean with standard deviation. There was no mention of age in one $(\mathrm{N}=1)$ study. Comparative evaluation of juvenile and adults was reported in two studies $(\mathrm{N}=2)$ and adolescent vs. adults in one study $(\mathrm{N}=1)$. Age groups of male and females subjects were managed separately in three studies $(\mathrm{N}=3)$. Age group of up to 15 years was considered in $19(\mathrm{~N}=19)$ studies and 15 years and above was considered in $20(\mathrm{~N}=20)$ studies. One study 
Table 1 Participant characteristics

\begin{tabular}{|c|c|c|c|c|c|c|c|c|c|c|c|c|c|}
\hline $\begin{array}{l}\text { Ref } \\
\text { no. }\end{array}$ & Sx & $M / F$ & Age & Mediators & Index T & Cont $\mathrm{T}$ & Site $s p$ & Rnd & Mal & $\begin{array}{l}\mathrm{N} \text { drug } \\
\mathrm{H}\end{array}$ & $\begin{array}{l}N \text { bone } \\
\text { Ls }\end{array}$ & $\begin{array}{l}\text { N Ging } \\
\text { inflm }\end{array}$ & $\begin{array}{l}\text { PD } \\
(<2 \mathrm{~mm})\end{array}$ \\
\hline 22 & 16 & $2 \mathrm{M}, 14 \mathrm{~F}$ & 18 to $24 y$ & $\mathrm{IL}-1 \beta$ & Mx and Md C & Md R or $L C$ & $\mathrm{D}$ & Y & Class I biMx & Y & NM & Y & NM \\
\hline 23 & 22 & $11 \mathrm{M}, 11 \mathrm{~F}$ & $14.4+\ldots 1.1 y$ & Leptin & $M \times C$ & Contr C & $\mathrm{D}$ & $\mathrm{N}$ & $\begin{array}{l}\text { Mx 1st PM } \\
\text { Extr cs }\end{array}$ & Y & Y & Y & Y \\
\hline 24 & 33 & $21 \mathrm{M}, 12 \mathrm{~F}$ & 10.8 to $30.9 y$ & IL-1 $\beta$, IL-1RA & $M \times C$ & IP Md C/Aj T & $\mathrm{D}$ & y & $\begin{array}{l}\text { Mx 1st PM } \\
\text { Extr cs }\end{array}$ & NM & NM & NM & NM \\
\hline 25 & 12 & $7 F, 5 \mathrm{M}$ & 13 to $17 y$ & OPG & $M \times C$ & Contr C & $\mathrm{D}$ & $\mathrm{N}$ & $\begin{array}{l}\text { Mx 1st PM } \\
\text { Extr cs }\end{array}$ & Y & Y & Y & Y \\
\hline 26 & 9 & $5 \mathrm{M}, 4 \mathrm{M}$ & 10 to $18 y$ & $I L-1 \beta, \beta G$ & $\begin{array}{l}\text { 1st Mo, 1st } \\
\text { PM, Cl }\end{array}$ & NM & $\mathrm{MP}$ and $\mathrm{MB}$ & $\mathrm{N}$ & RME CS & NM & NM & NM & NM \\
\hline 27 & $\begin{array}{l}12 \mathrm{SD}(6) \\
\operatorname{LD}(6)\end{array}$ & $\begin{array}{l}\text { SD: } 3 \text { M, } \\
3 \text { FLD-NM }\end{array}$ & $\begin{array}{l}\text { SD: } 11 \text { to } 18 y \\
\text { LD: } 19 \text { to } 27 y\end{array}$ & IL-1 $\beta,-6, I L-8$, TNF- $a$ & Mx 1st PM & Ant T & DB & N & $\begin{array}{l}\text { Mx 2nd PM } \\
\text { Extr cs }\end{array}$ & Y & Y & Y & Y \\
\hline 28 & 10 & $4 \mathrm{M}, 6 \mathrm{~F}$ & NM & TNF-a & C & NM & $\mathrm{D}$ & $\mathrm{N}$ & $\begin{array}{l}\text { Mx 1st PM } \\
\text { Extr cs }\end{array}$ & Y & Y & Y & Y \\
\hline 29 & 18 & $9 \mathrm{M}, 9 \mathrm{~F}$ & 16 to $19 y$ & IL-1 $\beta,-$ TNF- $a$ & $M \times C$ & NM & $M$ and $D$ & $\mathrm{~N}$ & 1st PM Extr cs & Y & Y & Y & Y \\
\hline \multirow[t]{2}{*}{30} & \multirow{2}{*}{$\begin{array}{l}30: 15 \\
J / 15 \mathrm{~A}\end{array}$} & \multirow{2}{*}{$\begin{array}{l}J: 7 \mathrm{M}, 9 \mathrm{~F} \\
\mathrm{~A}: 6 \mathrm{M}, 9 \mathrm{~F}\end{array}$} & J: $15.1 \pm 2.8 y$ & \multirow[t]{2}{*}{ RANKL, OPG } & \multirow[t]{2}{*}{$1 \mathrm{M} \times \mathrm{C}$} & \multirow{2}{*}{$\begin{array}{l}\text { Contr and } \\
\text { ant } C\end{array}$} & \multirow[t]{2}{*}{ D } & \multirow[t]{2}{*}{$\mathrm{N}$} & \multirow{2}{*}{$\begin{array}{l}\text { Mx 1st PM } \\
\text { Extr cs }\end{array}$} & \multirow[t]{2}{*}{ Y } & \multirow[t]{2}{*}{ Y } & \multirow[t]{2}{*}{ Y } & \multirow[t]{2}{*}{ Y } \\
\hline & & & A: $31 \pm 3.6 y$ & & & & & & & & & & \\
\hline 31 & 15 & $6 \mathrm{M}, 9 \mathrm{~F}$ & 15 to $19 y$ & IL-2, IL-6, IL-8 & $M \times C$ & NM & $M$ and $D$ & N & 1st PM Extr cs & Y & Y & Y & Y \\
\hline \multirow[t]{2}{*}{32} & \multirow[t]{2}{*}{10} & \multirow[t]{2}{*}{$4 \mathrm{M}, 6 \mathrm{~F}$} & $M-14.5 y$ & \multirow[t]{2}{*}{ RANKL, OPG } & \multirow[t]{2}{*}{$M \times C$} & \multirow{2}{*}{$\begin{array}{l}\text { Contr and } \\
\text { opposing C }\end{array}$} & \multirow[t]{2}{*}{ D } & \multirow[t]{2}{*}{ N } & \multirow{2}{*}{$\begin{array}{l}\text { Mx 1st PM } \\
\text { Extr cs }\end{array}$} & \multirow[t]{2}{*}{ Y } & \multirow[t]{2}{*}{ Y } & \multirow[t]{2}{*}{ Y } & \multirow[t]{2}{*}{ Y } \\
\hline & & & F 15.4 y & & & & & & & & & & \\
\hline 33 & 18 & $10 \mathrm{M}, 8 \mathrm{~F}$ & 8.9 to $13.8 \mathrm{y}$ & IL-1 $\beta, S P, P G E 2$ & 1st Mx/Md M & $\begin{array}{l}\text { Ant L or } \\
\text { R 1st M }\end{array}$ & $\mathrm{DB}$ and $\mathrm{DP}$ & $N$ & $\begin{array}{l}\text { Crowding in } 1 \\
\text { or both jaws }\end{array}$ & Y & Y & Y & Y \\
\hline \multirow[t]{2}{*}{34} & \multirow[t]{2}{*}{9} & \multirow[t]{2}{*}{$3 \mathrm{M}, 6 \mathrm{~F}$} & M: $21.3 \pm 2.8 \mathrm{y}$ & $I L-1 \beta, S P$ & $M \times C$ & Contr C & $\mathrm{D}$ & $N$ & Mx 1st PM & Y & Y & Y & Y \\
\hline & & & $F: 23.1 \pm 2.4 y$ & & & & & & & & & & \\
\hline 35 & 10 & $3 \mathrm{M}, 7 \mathrm{~F}$ & $\begin{array}{l}10 \text { y } 5 \mathrm{~m} \text { to } 30 \mathrm{y} \\
11 \mathrm{~m}\end{array}$ & IL-1 $\beta$, IL-1RA & $R$ and $L M x C$ & Md. R C & $M$ and $D$ & Y & $\begin{array}{l}\text { Mx 1st PM } \\
\text { Extr cs }\end{array}$ & NM & NM & NM & NM \\
\hline 36 & 10 & NM & 15 to $17 y$ & IL-8 & $M x$ and $M d C$ & NM & $M$ and $D$ & & 1st PM Extr cs & Y & Y & Y & Y \\
\hline 37 & 10 & $2 \mathrm{M}, 8 \mathrm{~F}$ & 18.4 to $22.5 \mathrm{y}$ & IL-1 $\beta, P G E 2$ & $\begin{array}{l}\text { Mx C (E1), } \\
\text { Contr Mx C } \\
\text { (E2) }\end{array}$ & Ant Md C & $\mathrm{D}$ & $\mathrm{N}$ & $\begin{array}{l}\text { All 1st PM } \\
\text { Extr cs }\end{array}$ & Y & Y & Y & Y \\
\hline 38 & 84 & $43 \mathrm{~J}(\mathrm{M})$ & $\mathrm{J}: 11 \pm 0.7 \mathrm{y}$ & PGE-2, IL-6, GMCSF & $M \times L I$ & Contr LI & $M$ and $D B$ & $\mathrm{~N}$ & Labial tipping & Y & Y & Y & Y \\
\hline & & $41 \mathrm{~A}(\mathrm{M})$ & A: $-24 \pm 1.6 y$ & & & & & & & & & & \\
\hline 39 & 9 & $5 \mathrm{M}, 4 \mathrm{~F}$ & 10 to $18 y$ & $\| L-1 \beta \beta G$ & $\begin{array}{l}\text { 1st Mo, 1st } \\
\text { PM, Cl }\end{array}$ & NM & $\mathrm{MP}$ and $\mathrm{MB}$ & $\mathrm{N}$ & RME cs & Y & Y & Y & Y \\
\hline 40 & 7 & $2 \mathrm{M}, 5 \mathrm{~F}$ & $\begin{array}{l}12 \text { y } 3 \mathrm{~m} \text { to } 16 y \\
3 \mathrm{~m}\end{array}$ & IL-1 $\beta, I L-1 R A, A I$ & $M \times C$ & Md C & $M$ and $D$ & NM & All 1st PM Extr & Y & NM & Y & NM \\
\hline 41 & 12 & $3 \mathrm{M}, 9 \mathrm{~F}$ & $14.4 \pm 0.9 y$ & TGF- $\beta$ & C & Ant $\mathrm{C} /$ Contr C & $\mathrm{D}$ & NM & $\begin{array}{l}\text { All 1st PM } \\
\text { Extr }\end{array}$ & Y & Y & Y & Y \\
\hline 42 & 12 & $3 \mathrm{M}, 9 \mathrm{~F}$ & $14.4 \pm 0.9 y$ & IL-1 $\beta, \| L-6$, TNF- $\alpha$, EGF, $\beta 2-\mu G$ & c & Ant $\mathrm{C} /$ Contr C & D & NM & All 1st PM Extr & Y & Y & Y & Y \\
\hline
\end{tabular}


Table 1 Participant characteristics (Continued)

\begin{tabular}{|c|c|c|c|c|c|c|c|c|c|c|c|c|c|}
\hline 19 & 10 & $5 \mathrm{M}, 5 \mathrm{~F}$ & $\begin{array}{l}\text { M: } 24.6 \pm 1.5 \text { y F: } \\
27.8 \pm 3.9 \text { y }\end{array}$ & $\mathrm{IL}-1 \beta, \mathrm{PGE}$ & $\begin{array}{l}\text { Mx LI, Mx } \\
\text { 1st PM }\end{array}$ & Contr $\mathrm{T}$ & $M B$ & N & $\begin{array}{l}\text { Buccal/labial } \\
\text { OTM }\end{array}$ & Y & Y & Y & Y \\
\hline 43 & 50 & $23 \mathrm{M}, 27 \mathrm{~F}$ & 13 to $20 y$ & IL-1 $\beta$, TNF-a, NO & $M \times I, M x C$ & NM & $M$ and $D$ & NM & Non Extr & Y & NM & Y & Y \\
\hline 44 & 10 & $5 \mathrm{M}, 5 \mathrm{~F}$ & 12 to $16 y$ & RANKL, OPG & $\begin{array}{l}\text { 1st PM } \\
\text { (quad 1) }\end{array}$ & 1st PM (quad 2) & D & NM & $\begin{array}{l}\text { 2nd Mx PM } \\
\text { Extr }\end{array}$ & Y & NM & Y & NM \\
\hline 45 & 22 & $7 \mathrm{M}, 15 \mathrm{~F}$ & 19 to $29 y$ & HSP70, RANKL & $M \times C$ & Contr C & & NM & Mx 1st PM Extr & Y & NM & Y & NM \\
\hline 46 & 12 & $6 \mathrm{M}, 6 \mathrm{~F}$ & 11 to $15 y$ & MMP-8, IL-1 $\beta$ & 1st M, Cl, C & NM & M & NM & NM & Y & Y & Y & Y \\
\hline 47 & 21 & NM & 12 to $20 y$ & $\begin{array}{l}\text { GM-CSF, IF-Y, IL-1 } 13, \text { IL-2, IL-4, IL-5, IL-6, } \\
\text { IL-8, IL-10 and TNFa, MMP-9, TIMP-1 } \\
\text { and 2, RANKL, OPG }\end{array}$ & $M \times C$ & 2nd M & $\mathrm{MB}$ and $\mathrm{DP}$ & NM & Mx 1st PM Extr & Y & Y & Y & Y \\
\hline 48 & 10 & $5 \mathrm{M}, 5 \mathrm{~F}$ & 22 to $29 y$ & RANK, OPG, OPN, TGF- $\beta 1$ & 1st M & Contr M & $\begin{array}{l}\text { Exp T: MB and } \\
\text { DB Cont T: MB } \\
\text { and ML }\end{array}$ & Y & NM & Y & NM & Y & Y \\
\hline 49 & 14 & $3 \mathrm{M}, 11 \mathrm{~F}$ & 12 to $28 y$ & $\begin{array}{l}\text { MMP-3, MMP-9, MMP-13, MIP-1 } \beta \text {, MCP- } \\
\text { 1, RANTES }\end{array}$ & $M \times C$ & NM & $M$ and $D$ & NM & Mx 1st PM Extr & Y & NM & NM & NM \\
\hline 50 & 18 & $8 \mathrm{M}, 10 \mathrm{~F}$ & 8.9 to $13.8 \mathrm{y}$ & IL-1 $\beta, S P, P G E 2$ & 1st M & Contr M & $M$ and $D$ & NM & $\begin{array}{l}\text { Crowding in } \\
M x \text { and } M d\end{array}$ & Y & Y & Y & Y \\
\hline 51 & 10 & NM & $16.3 \pm 2.5 y$ & $\mathrm{IL}-1 \beta$ & $M \times C$ & Md C & DB & NM & $\begin{array}{l}\text { Class II, all } \\
\text { 1st PM Extr }\end{array}$ & Y & Y & Y & Y \\
\hline 52 & 16 & $8 \mathrm{M}, 8 \mathrm{~F}$ & $16.6 \pm 2.4 y$ & IL-2, IL-6, IL-8 & $M \times C$ & Mx 2nd PM & DB & NM & Mx 1st PM Extr & Y & Y & Y & Y \\
\hline 53 & 20 & $\begin{array}{l}\text { C: } 3 \mathrm{M}, 7 \mathrm{~F} \\
\mathrm{EX}: 5 \mathrm{M}, 5 \mathrm{~F}\end{array}$ & 18 to $45 y$ & $\begin{array}{l}\text { CCL-2 (MCP1), CCL-3, CCL-5 (RANTES), } \\
\text { IL-8 (CXCL8), IL-1a, IL-1 } 13, \text { IL-6, TNF-a }\end{array}$ & $M \times C$ & Contr C & DB & Y & $\begin{array}{l}\text { Class II Div } 1 \\
\text { Mal With 1st } \\
\text { PM Extr }\end{array}$ & Y & Y & Y & Y \\
\hline 54 & 16 & $8 \mathrm{M}, 8 \mathrm{~F}$ & $16.6 \pm 2.4 y$ & TNF-a & $M \times C$ & Mx 2nd PM & DB & NM & Mx 1st PM Extr & Y & Y & Y & Y \\
\hline 55 & 17 & $9 \mathrm{M}, 8 \mathrm{~F}$ & 16 to $20 y$ & TNF- $\alpha, I L-1 \beta, I L-8$ & $M \times C$ & NM & $M$ and $D$ & NM & All 1st PM Extr & Y & Y & Y & Y \\
\hline 56 & 10 & $5 \mathrm{M}, 5 \mathrm{~F}$ & 15 y \pm 3 y $8 m$ & IL-1ß, IL-1RA & $M \times C$ & $\mathrm{Md} \mathrm{C/Aj} \mathrm{t}$ & D & NM & All 1st PM Extr & NM & NM & NM & NM \\
\hline 57 & 20 & $\begin{array}{l}10 \text { ado }(3 \mathrm{M}, 7 \mathrm{~F}) \\
10 \mathrm{~A}(4 \mathrm{M}, 6 \mathrm{~F})\end{array}$ & $\begin{array}{l}\text { Ado: } 14.4 \pm 1.43 \text { y } \\
\text { A: }-28.5 \pm 7.83 \text { y }\end{array}$ & RANKL, OPG, IL-1, IL-1RA, MMP-9 & MxI & Md I & Lab & NM & $\begin{array}{l}\text { Non Extr, } \\
3 \text { to } 6 \mathrm{~mm} \\
\text { I crowding }\end{array}$ & $N$ & $\mathrm{~N}$ & Y & $N$ \\
\hline 58 & $\begin{array}{l}24 \mathrm{HG}-14 \\
\mathrm{NHG}-10\end{array}$ & $\begin{array}{l}10 \mathrm{M}, 14 \mathrm{FHG}: \\
6 \mathrm{M}, 8 \mathrm{~F}\end{array}$ & $14.66 \pm 1.1 \mathrm{y}$ & $\begin{array}{l}\text { IL-2, IL-4, IL-6, IL-8, IL-10, GM-CSF, IFN- } \gamma \\
\text { TNFa, MCP-1, IP-10 }\end{array}$ & $\begin{array}{l}\text { Mx 1st M } \\
\text { and Mx } \\
\text { 1st PM }\end{array}$ & NM & $\mathrm{MB}$ and $\mathrm{DB}$ & $N$ & Non extr cs & Y & NM & Y & Y \\
\hline 59 & 9 & $4 \mathrm{M}, 5 \mathrm{~F}$ & 17.5 to $18.9 y$ & OPG, RANKL & $M \times C$ & NM & $M$ and $D$ & NM & All 4 Extr & Y & Y & Y & $Y$ \\
\hline
\end{tabular}


Table 2 Study characteristics

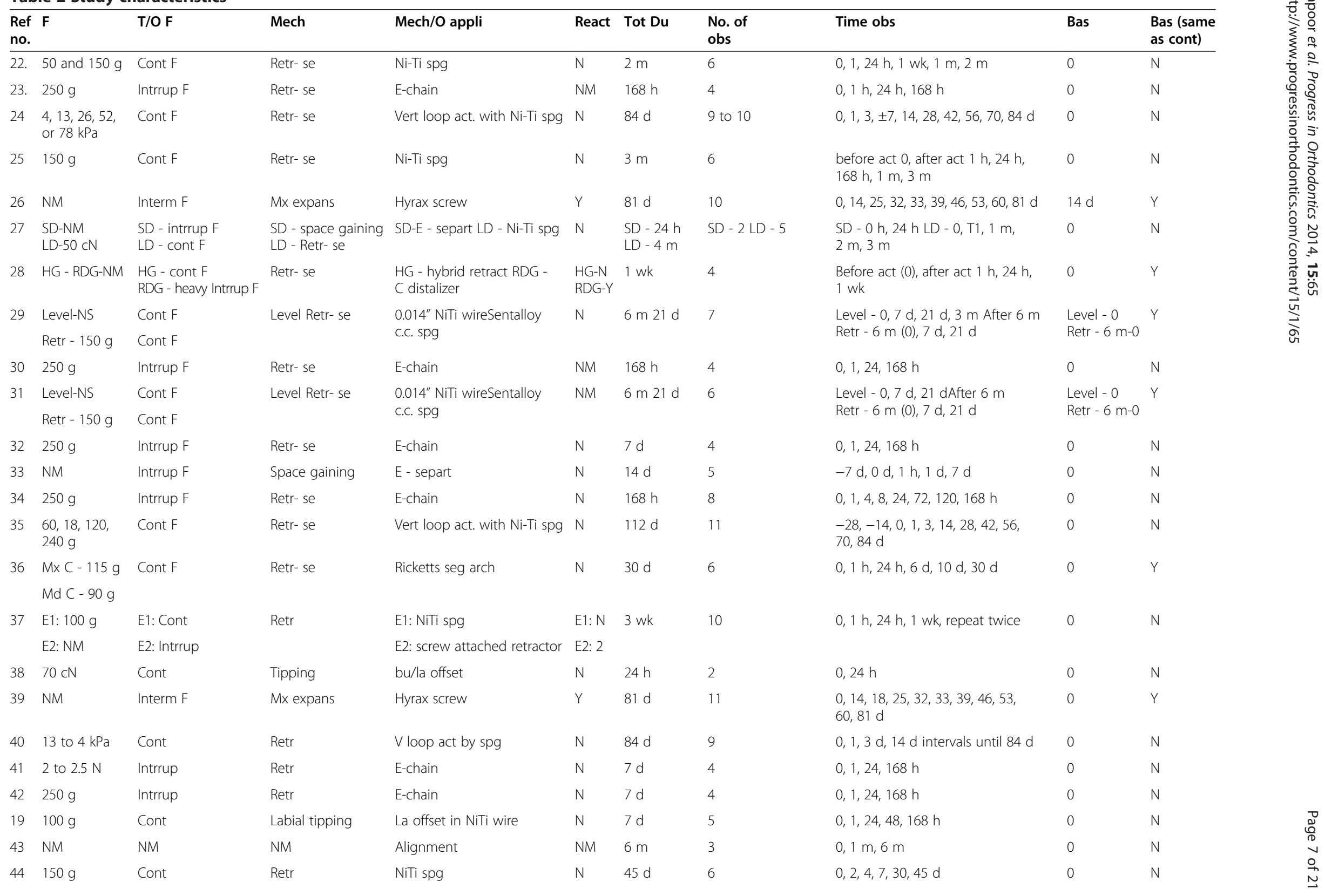


Table 2 Study characteristics (Continued)

\begin{tabular}{|c|c|c|c|c|c|c|c|c|c|c|}
\hline 45 & $130 \mathrm{~g}$ & Intrrup & Retr & E-chain & N & $24 \mathrm{~h}$ & 2 & $0,24 \mathrm{~h}$ & 0 & $\mathrm{~N}$ \\
\hline 46 & NM & NM & NM & Bracket placement & N & $3 \mathrm{~m}$ & 4 & $0,24 \mathrm{~h}, 1 \mathrm{wk}, 3 \mathrm{~m}$ & 0 & Y \\
\hline 47 & $100 \mathrm{~g}$ & Cont & Retr & NiTi c.c. spg & N & $42 \mathrm{~d}$ & 4 & $-10 w k, 0,4 h, 7 d, 42 d$ & 0 & $\mathrm{~N}$ \\
\hline 48 & NM & Intrrup & Space gain & Elastic separt & N & $7 d$ & 3 & $0,24 h, 7 d$ & 0 & $\mathrm{~N}$ \\
\hline 49 & $150 \mathrm{~g}$ & Cont & Retr & V - loop and NiTi c.c. spg & $\mathrm{N}$ & $87 d$ & 7 & $-7 \mathrm{~d}, 0,1 \mathrm{~h}, 24 \mathrm{~h}, 14,21,80 \mathrm{~d}$ & 0 & Y \\
\hline 50 & NM & Intrrup & Space gin & Elastic separt & $\mathrm{N}$ & $14 d$ & 6 & $-7 \mathrm{~d}, 0,1 \mathrm{~min}, 1 \mathrm{~h}, 1 \mathrm{~d}, 7 \mathrm{~d}$ & 0 & $\mathrm{~N}$ \\
\hline 51 & $120 \mathrm{~g}$ & Cont & Retr & NiTi c.c. spg & N & $21 d$ & 6 & 1 h, 24 h, 48 h, 168 h, 14 d, $21 d$ & $1 \mathrm{~h}$ & $\mathrm{~N}$ \\
\hline 52 & $150 \mathrm{~g}$ & Cont & Retr & Sentalloy c.c. spg & N & $3 \mathrm{~m}$ & 7 & $0,1,24,48 \mathrm{~h}, 7 \mathrm{~d}, 21 \mathrm{~d}, 3 \mathrm{~m}$ & 0 & $\mathrm{~N}$ \\
\hline 53 & $100 \mathrm{~g}$ & Cont F & Retr- se & $\mathrm{Ni}$-Ti c.C. spg & $\mathrm{N}$ & $28 \mathrm{~d}$ & 4 & $0,1 \mathrm{~d}, 7 \mathrm{~d}, 28 \mathrm{~d}$ & 0 & Y \\
\hline 54 & $150 \mathrm{~g}$ & Cont & Retr & Sentalloy c.c. spg & $\mathrm{N}$ & $3 \mathrm{~m}$ & 7 & $0,1,24,48 \mathrm{~h}, 7 \mathrm{~d}, 21 \mathrm{~d}, 3 \mathrm{~m}$ & 0 & $\mathrm{~N}$ \\
\hline 55 & NM & Cont & Alignment & 0.014 NiTi wire & $\mathrm{N}$ & $7 d$ & 8 & $0,1,2,3,4,5,6,7 d$ & 0 & Y \\
\hline 56 & $\begin{array}{l}13,26 \\
52 \mathrm{kPa}\end{array}$ & Cont & Retr & $V$ loop act by spg & $\mathrm{N}$ & $84 \mathrm{~d}$ & 9 & $0,1,3 \mathrm{~d}, 14 \mathrm{~d}$ intervals until $84 \mathrm{~d}$ & 0 & $\mathrm{~N}$ \\
\hline 57 & NM & Cont & Alignment & $\begin{array}{l}0.014-\mathrm{NiTi}, 0.016 \times 0.022 \\
\mathrm{NiTi}, 0.019 \times 0.025 \mathrm{NiTi}\end{array}$ & $\mathrm{N}$ & $20 w k$ & 6 & $0,3 w k, 6 w k, 12 w k, 2 w k$ & 0 & $\mathrm{~N}$ \\
\hline \multirow[t]{3}{*}{58} & \multirow[t]{3}{*}{ NM } & \multirow{3}{*}{$\begin{array}{l}\text { HG: band intrrup F } \\
\text { Bond - Cont F } \\
\text { NHG - Cont F }\end{array}$} & \multirow[t]{3}{*}{ Level } & \multirow[t]{3}{*}{$\begin{array}{l}0.014 \text { - in NiTi wire } 0.016 \times \\
0.022 \text {-in s.s. }\end{array}$} & \multirow[t]{3}{*}{$\mathrm{N}$} & \multirow[t]{3}{*}{$70 w k$} & \multirow[t]{3}{*}{$\begin{array}{l}\text { HG: band-3 HG; } \\
\text { bond-2 NHG-2 }\end{array}$} & HG: band - 18, 0, $52 \mathrm{wk}$ & $\begin{array}{l}\text { HG: band - } \\
18 \text { wk }\end{array}$ & \multirow[t]{3}{*}{ Y } \\
\hline & & & & & & & & HG: bond - 0,52 wk & Bond - 0 wk & \\
\hline & & & & & & & & $\mathrm{NHG}-0,52 \mathrm{wk}$ & NHG - 0 wk & \\
\hline 59 & $200 \mathrm{~g}$ & Cont & Retr & Sentalloy c.c. spg & N & $1 \mathrm{~m}$ & 5 & 0,1 h, 24 h, 168 h, $1 \mathrm{~m}$ & 0 & Y \\
\hline
\end{tabular}

Ref No. reference number, $F$ force, T/O type of, Mech mechanics, Mech/O mechanics of, appli appliance, React reactivation, Tot total, Du duration, $N$ number, obs observation, Bas baseline, min minutes, $g$ grams, Intrup interrupted, Cont continuous, interm intermittent, Retr retraction, se segmented, spg spring, E-chain elastomeric chain, NiTi nitinol, cont control, NM not mentioned, $y$ year, $d$ day, $m$ month, $h$ hour, Level leveling, separt separator, act activated, $H G$ headgear, NHG non-headgear, bu buccal, la labial, $H G$ hybrid retract, RDG rapid canine distalizer. 
Table 3 Oral hygiene regimen and assessment of gingival health

\begin{tabular}{|c|c|c|c|c|c|c|}
\hline Ref no. & Mu wsh & $\begin{array}{l}\text { Freq/O } \\
\text { Mu wsh/d }\end{array}$ & $\begin{array}{l}\text { Oral prophy } \\
\text { (pre t/t) }\end{array}$ & $\begin{array}{l}\text { Oral prophy } \\
\text { (every obser pt) }\end{array}$ & $\begin{array}{l}\text { Asses for ging and perio } \\
\text { inflam (pre } t / t)\end{array}$ & $\begin{array}{l}\text { At every } \\
\text { obser pt }\end{array}$ \\
\hline 22 & Chlorhex & Twice & NM & NM & $Y$ & $Y$ \\
\hline 23 & $\mathrm{~N}$ & NA & Y & Y & Y & At 0 and $168 \mathrm{~h}$ \\
\hline 24 & NM & NM & Y & Y & Y & Y \\
\hline 25 & NM & NA & Y & Y & $\mathrm{N}$ & $\mathrm{N}$ \\
\hline 26 & Chlorhex & Twice & Y & NM & Y & Y \\
\hline 27 & Chlorhex & Twice & Y & Y & Y & Y \\
\hline 28 & NM & NA & NM & NM & Y & NM \\
\hline 29 & NM & NA & NM & NM & NM & NM \\
\hline 30 & NM & NA & Y & Y & Y & Y \\
\hline 31 & NM & NA & NM & NM & NM & NM \\
\hline 32 & NM & NA & Y & Y & Y & Y \\
\hline 33 & Chlorhex & Twice & Y & Y & Y & Y \\
\hline 34 & NM & NA & Y & Y & Y & Y \\
\hline 35 & Chlorhex & Twice & Y & Y & Y & Y \\
\hline 36 & NM & NM & Y & Y & Y & Y \\
\hline 37 & NM & NM & NM & NM & Y & Y \\
\hline 38 & NM & NM & Y & Y & NM & NM \\
\hline 39 & Chlorhex & Twice & Y & NM & Y & Y \\
\hline 40 & Chlorhex & Twice & Y & Y & Y & Y \\
\hline 41 & NM & NM & NM & NM & Y & Y \\
\hline 42 & NM & NM & NM & NM & Y & Y \\
\hline 19 & Chlorhex & NM & NM & NM & Y & \\
\hline 43 & NM & NM & Y & Y & Y & Y \\
\hline 44 & Chlorhex & Twice & Y & Y & Y & Y \\
\hline 45 & NM & NM & Y & Y & NM & NM \\
\hline 46 & NM & NM & Y & $\mathrm{Y}(6 \mathrm{wk}, 3 \mathrm{~m})$ & Y & Y \\
\hline 47 & NM & NM & NM & NM & Y & Y \\
\hline 48 & NM & NM & Y & Y & Y & Y \\
\hline 49 & Chlorhex & Twice & Y & Y & NM & NM \\
\hline 50 & NM & NM & Y & N & Y & Y \\
\hline 51 & NM & NM & NM & NM & NM & NM \\
\hline 52 & NM & NM & Y & Y & Y & NM \\
\hline 53 & NM & NM & Y & Y & Y & NM \\
\hline 54 & NM & NM & Y & Y & Y & NM \\
\hline 55 & NM & NM & NM & NM & NM & NM \\
\hline 56 & Chlorhex & Twice & Y & Y & Y & Y \\
\hline 57 & NM & & Y & NM & NM & NM \\
\hline 58 & NM & NA & Y & NM & Y & Y \\
\hline 59 & NM & & Y & Y & Y & Y \\
\hline
\end{tabular}

Ref No. reference number, Mu mouth, wsh wash, Freq/O frequency of, $d$ day, prophy prophylaxis, $t / t$ treatment, obser observation, $p t$ point, Asses assessment, ging gingival, perio periodontal, inflam inflammation, chlorhex chlorhexidine, $Y$ yes, $N M$ not mentioned, $N$ no, $h$ hour. 
Table 4 GCF characteristics

\begin{tabular}{|c|c|c|c|c|c|c|c|c|c|c|c|c|c|}
\hline $\begin{array}{l}\text { Ref } \\
\text { no. }\end{array}$ & Time & Temp & Humd & Site $s p$ & $\begin{array}{l}\text { Inser } \\
\text { (in mm) }\end{array}$ & $\begin{array}{l}\text { Rep } \\
\text { meas }\end{array}$ & $\begin{array}{l}1 / 0 \\
\text { meas }\end{array}$ & $\begin{array}{l}\text { Meth/O } \\
\text { coll }\end{array}$ & $\begin{array}{l}\mathrm{Du} / \mathrm{O} \\
\text { meas }\end{array}$ & $\begin{array}{l}\text { Temp } \\
\text { of sto }\end{array}$ & $\begin{array}{l}\text { Meth/O } \\
\text { meas }\end{array}$ & $\begin{array}{l}\text { Anal } \\
\text { meth }\end{array}$ & Prot conc \\
\hline 22 & NM & NM & NM & D & $1 \mathrm{~mm}$ & 2 & $90 \mathrm{~s}$ & PP & $30 \mathrm{~s}$ & $-80^{\circ} \mathrm{C}$ & PT8000 & ELISA & $\mathrm{pg} / \mathrm{mg}$ \\
\hline 23 & Y & NM & NM & D & $1 \mathrm{~mm}$ & 4 & $60 \mathrm{~s}$ & PP & $30 \mathrm{~s}$ & $-80^{\circ} \mathrm{C}$ & PT8000 & ELISA & $\mathrm{pg} / \mathrm{ml}$ \\
\hline 24 & NM & NM & NM & D & NM & 2 & NM & NM & NM & NM & NM & ELISA & NM \\
\hline 25 & NM & NM & NM & D & $1 \mathrm{~mm}$ & N & NA & PP & $30 \mathrm{~s}$ & $-80^{\circ} \mathrm{C}$ & PT8000 & ELISA & $\mathrm{pg} / \mu \mathrm{l}$ \\
\hline 26 & NM & Y & Y & M & NM & $\mathrm{N}$ & NA & PP & $30 \mathrm{~s}$ & NM & PT6000 & ELISA & $\mathrm{pg} / 30 \mathrm{~s}$ \\
\hline 27 & NM & NM & NM & DB & $1 \mathrm{~mm}$ & $N$ & NA & PP & $30 \mathrm{~s}$ & $-20^{\circ} \mathrm{C}$ & PT8000 & $M B-I A$ & $\mathrm{pg} / \mu \mathrm{l}$ \\
\hline 28 & Y & NM & NM & D & $1 \mathrm{~mm}$ & 4 & $60 \mathrm{~s}$ & PP & $30 \mathrm{~s}$ & $-80^{\circ} \mathrm{C}$ & Elec scale & ELISA & $\mathrm{pg} / \mu \mathrm{l}$ \\
\hline 29 & Y & NM & NM & $M$ and $D$ & $1 \mathrm{~mm}$ & N & NA & PP & $30 \mathrm{~s}$ & $-20^{\circ} \mathrm{C}$ & PT8000 & $\mid A$ & $\mathrm{pg}$ \\
\hline 30 & NM & NM & NM & D & $1 \mathrm{~mm}$ & 2 & $60 \mathrm{~s}$ & PP & $60 \mathrm{~s}$ & $-30^{\circ} \mathrm{C}$ & PT8000 & ELISA & $\mathrm{pg} / \mu \mathrm{l}$ \\
\hline 31 & Y & NM & NM & $M$ and $D$ & NM & N & NA & PP & $30 \mathrm{~s}$ & $-20^{\circ} \mathrm{C}$ & PT8000 & IA & $\mathrm{pg}$ \\
\hline 32 & NM & NM & NM & D & $1 \mathrm{~mm}$ & 1 & $60 \mathrm{~s}$ & PP & $60 \mathrm{~s}$ & $-30^{\circ} \mathrm{C}$ & PT8000 & ELISA & $\mathrm{pg} / \mu \mathrm{l}$ \\
\hline 33 & NM & NM & NM & $\mathrm{DB}$ and $\mathrm{DP}$ & $1 \mathrm{~mm}$ & NM & NA & DuFM & $20 \mathrm{~s}$ & $-70^{\circ} \mathrm{C}$ & NM & ELISA & $\mathrm{pg} / 20$-s samp \\
\hline 34 & NM & NM & NM & D & $1 \mathrm{~mm}$ & 2 & NM & PP & $60 \mathrm{~s}$ & $-30^{\circ} \mathrm{C}$ & PT8000 & ELISA & $\mathrm{pg} / \mathrm{\mu g}$ \\
\hline 35 & NM & NM & NM & $M$ and $D$ & $1 \mathrm{~mm}$ & 1 & $60 \mathrm{~s}$ & PP & $30 \mathrm{~s}$ & $-70^{\circ} \mathrm{C}$ & NM & ELISA & $\begin{array}{c}\mathrm{mg} / \mathrm{l} \text { - tot prot, } \\
\text { IL-1-ng/g IL-1RA- } \mu \mathrm{g} / \mathrm{g}\end{array}$ \\
\hline 36 & NM & NM & NM & $M$ and $D$ & $1 \mathrm{~mm}$ & 1 & $60 \mathrm{~s}$ & PP & $30 \mathrm{~s}$ & $-70^{\circ} \mathrm{C}$ & Elect scale & ELISA & $\mathrm{pg} / \mathrm{ml}$ \\
\hline 37 & Y & Y & Y & D & $1 \mathrm{~mm}$ & 4 & $1 \mathrm{~min}$ & PP & $30 \mathrm{~s}$ & $-70^{\circ} \mathrm{C}$ & NM & ELISA & $\mathrm{pg} / \mathrm{\mu g}$ \\
\hline 38 & NM & Y & NM & $\mathrm{MB}$ and $\mathrm{DB}$ & $1 \mathrm{~mm}$ & N & $\mathrm{N}$ & PP & $30 \mathrm{~s}$ & $-80^{\circ} \mathrm{C}$ & PT6000 & RIA & $\mathrm{pg} / \mu \mathrm{l}$ \\
\hline 39 & NM & Y & Y & M & NM & N & NA & PP & $30 \mathrm{~s}$ & NM & PT6000 & ELISA & pg/30-s GCF \\
\hline 40 & NM & NM & NM & $M$ and $D$ & $1 \mathrm{~mm}$ & 1 & $1 \mathrm{~min}$ & PP & $30 \mathrm{~s}$ & $-70^{\circ} \mathrm{C}$ & NM & ELISA & $\begin{array}{l}\text { Tot prot - mg/l, IL-1 } \beta \\
(\mathrm{ng} / \mathrm{g}), \mathrm{LL}-1 \mathrm{RA}(\mu \mathrm{g} / \mathrm{g})\end{array}$ \\
\hline 41 & NM & NM & NM & D & $1 \mathrm{~mm}$ & 1 & $1 \mathrm{~min}$ & PP & $30 \mathrm{~s}$ & $-30^{\circ} \mathrm{C}$ & PT & $\begin{array}{c}\text { ELISA, EP, } \\
\text { WB }\end{array}$ & $\mathrm{pg} / \mu \mathrm{g}$ \\
\hline 42 & NM & NM & NM & D & $1 \mathrm{~mm}$ & 1 & $1 \mathrm{~min}$ & PP & $30 \mathrm{~s}$ & $-30^{\circ} \mathrm{C}$ & PT & ELISA & $\mathrm{pg} / \mu \mathrm{g}$ \\
\hline 19 & Y & Y & y & $\mathrm{MB}$ & $1 \mathrm{~mm}$ & N & $\mathrm{N}$ & PP & $30 \mathrm{~s}$ & $-80^{\circ} \mathrm{C}$ & PT6000 & RIA & pg \\
\hline 43 & NM & NM & NM & $M$ and $D$ & NM & NM & NM & PP & $30 \mathrm{~s}$ & NM & PT8000 & ELISA & NM \\
\hline 44 & NM & NM & NM & D & NM & $N$ & $\mathrm{~N}$ & PP & $30 \mathrm{~s}$ & $-80^{\circ} \mathrm{C}$ & PT8000 & ELISA & $\mathrm{pg} / \mu \mathrm{l}$ \\
\hline 45 & NM & NM & NM & D & $2 \mathrm{~mm}$ & 1 & $2 \min$ & PP & $1 \mathrm{~min}$ & $-20^{\circ} \mathrm{C}$ & NM & $\begin{array}{l}\text { ELISA, WB, } \\
\text { SDS-PAGE }\end{array}$ & NM \\
\hline 46 & NM & NM & NM & M & NM & $N$ & $\mathrm{~N}$ & FP & $3 \mathrm{~min}$ & $-30^{\circ} \mathrm{C}$ & NM & ELISA & $\begin{array}{l}\text { IL-1 } \beta ; p g / m l, \\
\text { MMP-8: ng/ml }\end{array}$ \\
\hline 47 & NM & NM & NM & $\mathrm{MB}$ and $\mathrm{DP}$ & NM & N & $\mathrm{N}$ & PP & $30 \mathrm{~s}$ & NM & PT8000 & LMAT & $\mathrm{pg} / \mathrm{ml}$ \\
\hline 48 & NM & NM & NM & $\begin{array}{l}\text { ExpT: } M B \text { and } D B \\
\text { Cont T: } M B \text { and } M L\end{array}$ & $1 \mathrm{~mm}$ & $\mathrm{~N}$ & $\mathrm{~N}$ & PP & $30 \mathrm{~s}$ & NM & PT8000 & ELISA & $\begin{array}{c}\text { Cyt conc - pg/ } / \text { lTot } \\
\text { prot (pg) }\end{array}$ \\
\hline 49 & NM & NM & NM & $M$ and $D$ & $1 \mathrm{~mm}$ & $N$ & $\mathrm{~N}$ & PP & NM & $-80^{\circ} \mathrm{C}$ & PT8000 & $M B-I A$ & $\mathrm{pg} / \mathrm{site}$ \\
\hline 50 & NM & NM & NM & $\mathrm{MB}$ and $\mathrm{DB}$ & $1 \mathrm{~mm}$ & $\mathrm{~N}$ & $\mathrm{~N}$ & FM & $20 \mathrm{~s}$ & $-70^{\circ} \mathrm{C}$ & NM & ELISA & $\mathrm{pg} / 20 \mathrm{~s}$ \\
\hline 51 & Y & Y & Y & DB & NM & N & $\mathrm{N}$ & $\mathrm{FP}$ & $3 \mathrm{~min}$ & $-70^{\circ} \mathrm{C}$ & NM & ELISA & $\mathrm{pg} / \mu \mathrm{l}$ \\
\hline 52 & Y & NM & NM & DB & NM & 1 & $1 \mathrm{~min}$ & PP & $30 \mathrm{~s}$ & $-20^{\circ} \mathrm{C}$ & PT8000 & ELISA & $\mathrm{pg} / \mu \mathrm{l}$ \\
\hline 53 & Y & NM & NM & DB & $1 \mathrm{~mm}$ & 0 & NA & FP & $10 \mathrm{~s}$ & NM & PT8000 & CPA & $\mathrm{pg} / \mu \mathrm{l}$ \\
\hline 54 & $Y$ & NM & NM & $\mathrm{DB}$ & NM & 1 & $1 \mathrm{~min}$ & PP & $30 \mathrm{~s}$ & $-20^{\circ} \mathrm{C}$ & РT8000 & $\mid A$ & $\mathrm{pg} / \mathrm{\mu l}$ \\
\hline 55 & Y & NM & NM & NM & $1 \mathrm{~mm}$ & N & $\mathrm{N}$ & PP & $30 \mathrm{~s}$ & $-20^{\circ} \mathrm{C}$ & PT8000 & $\mathrm{IA}$ & $\mathrm{pg}$ \\
\hline 56 & NM & NM & NM & D & $1 \mathrm{~mm}$ & 1 & $1 \mathrm{~min}$ & PP & $30 \mathrm{~s}$ & $-70^{\circ} \mathrm{C}$ & NM & ELISA & $\begin{array}{l}\text { Tot prot - mg/l, IL-1 } \\
(\mathrm{ng} / \mathrm{g}), \mathrm{LL}-1 \mathrm{RA}(\mu \mathrm{g} / \mathrm{g})\end{array}$ \\
\hline
\end{tabular}


Table 4 GCF characteristics (Continued)

\begin{tabular}{|c|c|c|c|c|c|c|c|c|c|c|c|c|c|}
\hline 57 & NM & NM & NM & $\mathrm{DL}$ & $1 \mathrm{~mm}$ & $\mathrm{~N}$ & $\mathrm{~N}$ & PP & $30 \mathrm{~s}$ & $-80^{\circ} \mathrm{C}$ & NM & QAK & $\mathrm{pg} / \mathrm{ml}$ \\
\hline 58 & NM & NM & NM & $\mathrm{MB}$ and $\mathrm{DB}$ & NM & 2 & NA & PP & $30 \mathrm{~s}$ & $-70^{\circ} \mathrm{C}$ & PT6000 & $\mathrm{BHCA}$ & $\mathrm{pg} / \mathrm{ml}$ \\
\hline 59 & NM & NM & NM & $M$ and $D$ & $1 \mathrm{~mm}$ & 1 & $1 \mathrm{~min}$ & PP & $30 \mathrm{~s}$ & $-70^{\circ} \mathrm{C}$ & $\begin{array}{c}\text { Precisa } 62 \\
\text { A }\end{array}$ & ELISA & $\mathrm{pmol} / \mathrm{l}$ \\
\hline
\end{tabular}

Ref No. reference number, Humd humidity, sp specification, Inser insertion, $M B$ mesio-buccal, $M L$ mesio-lingual, $D P$ disto-palatal sites, $D B$ disto-buccal sites, $M$ mesial, $D$ distal, $N M$ not mentioned, $N$ no, Y yes, $P P$ periopaper, $P T$ periotron, FP filter paper strips, FM Durapore filter membrane, WB Western blot, ELISA enzyme linked immune sorbent assay, SDS-PAGE sodium-dodecyl sulfate polyacrylamide gel electrophoresis, RIA radio-IA, I/O interval of, Meth/O method of, coll collection, meas measurement, Du/O duration of, Temp temperature, sto storage, Anal analysis, Prot protein, conc concentration, $p g$ picogram, $m g$ microgram, $\mathrm{ml} \mathrm{milliliter,} \mu \mathrm{lmicroliter}$, GCF gingival crevicular fluid, tot total, $g$ gram, $n g$ nanogram, $s$ seconds, min minutes, ${ }^{\circ} \mathrm{C}$ degree Celsius, elect electronic, IA immunoassay, EP electrophoresis, $s P$ spectrophotometry, $A r$ array, $A$ assay, $M B$ multiplex bead, LMAT Luminex multianalyte technology, BHCA Bio-Plex human cytokine assay, CPA custom protein array, QAK Quantibody Ar kit, DuFM Durapore filter membrane.

$(\mathrm{N}=1)$ considered a large age group interval of 18 to 45 years [53].

\section{Mediators of orthodontic tooth movement}

Cytokines, receptors and their antagonists included in the review have been listed in Table 1 . The cytokines have been studied singularly or in combination with other mediators. The most often studied cytokines in tooth movement are IL- $1 \beta(\mathrm{N}=21)$, TNF- $\alpha(\mathrm{N}=10)$, IL-8 $(\mathrm{N}=8)$, IL-6 $(\mathrm{N}=8)$, IL-2 $(\mathrm{N}=4)$, IL-4, IL-10 ( $\mathrm{N}=2)$, IL-1, IL-5, IL-1 $\alpha(\mathrm{N}=1), \mathrm{OPN}(\mathrm{N}=1)$, and RANKL $(\mathrm{N}=7)$.

Receptors and their antagonists have been studied in the frequency of OPG $(\mathrm{N}=8)$, IL 1RA $(\mathrm{N}=5)$, and RANK (N $=1)$. Chemokines have been studied in the order of monocyte chemoattractant protein (MCP)-1 $(\mathrm{N}=3)$, RANTES $(\mathrm{N}=2)$, and IP-10 $(\mathrm{N}=1)$. Studies related to growth factors were $\operatorname{GMCSF}(\mathrm{N}=3)$, TGF- $\beta(\mathrm{N}=2)$, and IFN- $\gamma(\mathrm{N}=2)$ and there was only one $(\mathrm{N}=1)$ study on Leptin.

\section{Time period and observation intervals}

The total time duration for studies exhibited large variation from as low as $24 \mathrm{~h}$ to as high as 70 weeks. Studies were performed for a duration of $24 \mathrm{~h}(\mathrm{~N}=3), 1$ week $(\mathrm{N}=10)$, 2 weeks $(\mathrm{N}=2), 3$ weeks $(\mathrm{N}=2)$, approximately 1 month $(\mathrm{N}=5), 2$ months $(\mathrm{N}=1)$, approximately 3 months $(\mathrm{N}=10)$, 4 months $(\mathrm{N}=2), 5$ months $(\mathrm{N}=1)$, and 6 months and above $(\mathrm{N}=4)$.

GCF collection was done at multiple observation times ranging from a maximum of 11 times $(\mathrm{N}=2)$ to minimum at 2 times $(\mathrm{N}=4)$. Nine studies $(\mathrm{N}=9)$ used 4 observation times; eight $(\mathrm{N}=8)$ studies used 6 observation times; four $(\mathrm{N}=4)$ studies used 2, 5, and 7 observation times; three $(\mathrm{N}=3)$ studies used 3,9 , and 10 observation times; and two $(\mathrm{N}=2)$ studies used 8 observation times.

Thirty-seven $(\mathrm{N}=37)$ studies took observation point before activation as 'zero' or baseline. The protocol followed for GCF collection was $0,1 \mathrm{~h}, 24 \mathrm{~h}$, and 7 days $(\mathrm{N}=14)$ and of $0,1 \mathrm{~h}$, and $24 \mathrm{~h}(\mathrm{~N}=15)$. An additional observation point at 14 days $(\mathrm{N}=7)$ and 21 days $(\mathrm{N}=6)$ was also considered. An internal control (baseline levels) was considered in $\mathrm{N}=12$ studies while other studies $(\mathrm{N}=27)$ took an external control that was either contralateral or antagonistic tooth or tooth other than experimental tooth.

\section{Study design}

\section{Mechanics of force application}

Twenty-seven studies considered retraction of canine $(\mathrm{N}=27)$ which included NiTi coil spring $(\mathrm{N}=18)$, vertical loop $(\mathrm{N}=5)$, screw-based retractors $(\mathrm{N}=3)$, and segmental mechanics $(\mathrm{N}=14)$. Other methods of force applications were separators $(\mathrm{N}=4)$, expansion with hyrax screw $(\mathrm{N}=2)$, labial tipping with offsets in wire $(\mathrm{N}=2)$, bracket placement $(\mathrm{N}=1)$, and leveling of arches $(\mathrm{N}=5)$.

\section{Type of force application}

Twenty-six studies $(\mathrm{N}=26)$ used continuous force and 12 $(\mathrm{N}=12)$ employed interrupted force. There was no mention of type of force in two $(\mathrm{N}=2)$ studies. Four studies used continuous and interrupted force on different index teeth $(\mathrm{N}=4)$.

\section{Levels of force}

Twenty-eight studies $(\mathrm{N}=28)$ mentioned levels of force applied while eleven studies $(\mathrm{N}=11)$ have no mention of it. One-hundred fifty-gram force was used in eight studies $(\mathrm{N}=8)$, followed by $250 \mathrm{~g}(\mathrm{~N}=5)$ and $100 \mathrm{~g}(\mathrm{~N}=4)$. One study $(\mathrm{N}=1)$ each employed 50, 120, 130, and $200 \mathrm{~g}$; $70 \mathrm{cN}$; and 2 to $2.5 \mathrm{~N}$ of force. A range of force was applied from 4 to $13 \mathrm{kPa}(\mathrm{N}=1), 90$ to $115 \mathrm{~g}(\mathrm{~N}=1)$, and $4,13,26,52$, or $78 \mathrm{kPa}$ in $\mathrm{N}=2$ studies. Force reactivation was considered in three $(\mathrm{N}=3)$ studies to compare continuous and interrupted force.

\section{Oral hygiene regimen and gingival health assessment}

Professional oral prophylaxis was performed before treatment $(\mathrm{N}=28)$ and at every observation point $(\mathrm{N}=23)$ but was not mentioned in the remaining studies. Oral hygiene regimen with recommendation of chlorhexidine mouthwash was mentioned in 11 studies $(\mathrm{N}=11)$ and its frequency $(\mathrm{N}=10)$ studies. Indices for assessment of gingival and periodontal health were employed before the treatment $(\mathrm{N}=30)$ and at every observation point in $\mathrm{N}=24$ studies.

\section{GCF characteristics}

The GCF samples were collected at a particular time of the day $(\mathrm{N}=11)$, preferably 9 AM to $12 \mathrm{PM}$ and early morning $(\mathrm{N}=2)$. Twenty-eight $(\mathrm{N}=28)$ studies had no mention of 
Table 5 Result characteristics

\begin{tabular}{|c|c|c|c|c|c|c|c|c|c|}
\hline $\begin{array}{l}\text { Ref } \\
\text { no. }\end{array}$ & Mediators & Stats analy appld & Confd & $\begin{array}{l}\text { Drop } \\
\text { outs }\end{array}$ & Up/down reg & $\mathrm{Pk}$ & Sec outcm & $r$ & Stat sign readings \\
\hline \multirow[t]{2}{*}{22.} & \multirow[t]{2}{*}{$\mathrm{IL}-1 \beta$} & \multirow[t]{2}{*}{$\begin{array}{l}\text { ANOVA and Friedman } \\
\text { and paired } t\end{array}$} & \multirow[t]{2}{*}{ Y } & \multirow[t]{2}{*}{ NM } & \multirow[t]{2}{*}{ Inc } & \multirow[t]{2}{*}{$24 \mathrm{~h}, 2 \mathrm{~m}$} & $\begin{array}{l}\text { 1. Mean tot prot conc - } \\
12 \mathrm{mg} / \mathrm{ml}\end{array}$ & \multirow[t]{2}{*}{$\begin{array}{l}\text { C mov with less pain and inflam } \\
\text { with } 50 \mathrm{~g} \text { than with } 150 \mathrm{~g} \text { of } \mathrm{F}\end{array}$} & \multirow[t]{2}{*}{$\begin{array}{l}\text { Inc at } 24 \mathrm{~h} \text { and } 2 \mathrm{~m} \text { in } 150 \mathrm{~g} \mathrm{~F} \text { compd } \\
\text { to cont }\end{array}$} \\
\hline & & & & & & & $\begin{array}{l}\text { 2. VAS score of } 150 \mathrm{~g}> \\
50 \mathrm{~g} \text { at } 24 \mathrm{~h}\end{array}$ & & \\
\hline 23. & Leptin & Wilcoxon, Friedman & NM & NM & Dec & $168 \mathrm{~h}$ & NM & NM & $\mathrm{b} / \mathrm{w}$ bas and $168 \mathrm{~h}$ in $\exp \mathrm{T}$ \\
\hline 24 & $\begin{array}{l}\text { IL-1 } \beta, \\
\text { IL-1RA }\end{array}$ & ANCOVA & Y & Y & Fluct & NM & $\begin{array}{l}\text { For same stress and grw status, } \\
\text { max diff in speed were 4.2:1 } \\
\text { for } 13 \mathrm{kPa} \text { in growers and 4.8:1 } \\
\text { for } 26 \mathrm{kPa} \text { in Nn-growers }\end{array}$ & $\begin{array}{l}\text { Higher speeds of T move sign } \\
\text { assoc with gen type } 2 \text { at IL-1 } \beta \\
(+3,954) \text {, higher Al, and lower } \\
\text { IL-1RA in GCF }\end{array}$ & NM \\
\hline 25 & OPG & $\begin{array}{l}\text { Shapiro Wilk's } \\
\text { Normality, Wilcoxon, } \\
\text { Friedman, Z }\end{array}$ & NM & NM & Dec & NM & NM & NM & $\begin{array}{l}\text { Dec at } 1 \text { h, } 24 \text { h, } 168 \text { h, } 1 \text { m, } 3 \text { m } \\
\text { compd with bas }\end{array}$ \\
\hline 26 & $\mathrm{IL}-1 \beta, \beta \mathrm{G}$ & 1 -tailed paired Student $t$ & Y & NM & Inc & $\begin{array}{l}\text { M-010 } \\
\text { PM-07 Cl-08 }\end{array}$ & $\begin{array}{l}\beta G \text { sign inc for } \mathrm{M}-07 \text { to } 010 \\
\text { PM-07,08,010 Cl - 06, } 07,010 \\
\text { and dec at O2 for M, PM, Cl }\end{array}$ & $\begin{array}{l}\text { Stronger } F \text { cause higher levels } \\
\text { of both } I L-1 \beta \text { and } \beta G\end{array}$ & $\begin{array}{l}\text { IL-1 } \beta \text { sign inc for } \mathrm{M}-\mathrm{O} 5 \text { to } \mathrm{O} 10 \\
\text { for PM-O6 to } 010 \text {. For } \mathrm{Cl}-04,06,07, \\
09,010 \text { and dec at } \mathrm{O} 2 \text { for } \mathrm{M}, \mathrm{PM}, \mathrm{Cl}\end{array}$ \\
\hline 27 & $\begin{array}{l}\text { IL-1 } \beta, \text { IL-6, } \\
\text { IL-8, TNF- } \alpha\end{array}$ & $\begin{array}{l}\text { Mann-Whitney, } \\
\text { Kruskal-Wallis }\end{array}$ & Y & NM & $\operatorname{lnc}$ & $\begin{array}{l}S D-24 h \\
L D-T 1\end{array}$ & NM & NM & $\begin{array}{l}\text { In SD IL-1 } \beta \text {, IL-8, TNF- } \alpha \text { inc, In LD } \\
\text { inc of IL-8 at T1 }\end{array}$ \\
\hline 28 & TNF-a & $\begin{array}{l}\text { Intergrp - Mann-Whitney } \\
\text { Ulntragrp - Wilcoxon } \\
\text { signed rank }\end{array}$ & NM & NM & Inc & $24 \mathrm{~h}$ & $\begin{array}{l}\mathrm{Pl}, \mathrm{PD}, \mathrm{BOP} \text { sign higher in } \mathrm{HG} \\
\mathrm{GCF} \text { vol at } 1 \mathrm{~h} \text { and } 24 \mathrm{~h} \text { in } \\
\text { the RDG }>\mathrm{HG}\end{array}$ & NM & $\begin{array}{l}\mathrm{HG} \text { - a stat sign dec at } 1 \mathrm{wk} \text { compd to } 24 \mathrm{~h} \text {. } \\
\mathrm{RDG} \text { - inc at } 1 \mathrm{~h} \text { stat sign compd to } \\
\text { initial value. Inc in RDG at } 1 \mathrm{~h}>\mathrm{HG}\end{array}$ \\
\hline 29 & IL-1 $\beta,-T N F-a$ & $\begin{array}{l}\text { 1-way paired } t \\
\text { Mann-Whitney } U\end{array}$ & NM & NM & $\operatorname{lnc}$ & $7 d, 21 d$ & $\begin{array}{l}\text { GCF vol inc on } 7 d \text { and } 21 d \\
\text { of level and retr }\end{array}$ & NM & $\begin{array}{l}\text { TNF - a diff b/w } 3 \text { m (level) and } \\
6 \mathrm{~m} \text { (bf retr) }\end{array}$ \\
\hline 30 & RANKL, OPG & $\begin{array}{l}\text { 3-way analysis of variance } \\
\text { Tukey's honest sign diff }\end{array}$ & Y & NM & $\begin{array}{l}\text { RANKL- Inc } \\
\text { OPG- Dec }\end{array}$ & $24 \mathrm{~h}$ & $\begin{array}{l}\text { avg amt of TM for J > A after } \\
168 \mathrm{~h} \text { mean vol of GCF in A } \\
\text { sign lower than J }\end{array}$ & GCF vol correl with inflam state & $\begin{array}{l}\text { RANKL at } 24 \mathrm{~h} \text { - sign inc levels both } \\
\text { in J \& A. RANKL and OPG in } \mathrm{A}<\mathrm{J} \\
\text { OPG at } 24 \mathrm{~h} \text { sign dec levels both in J \& } \mathrm{A} \text {. } \\
\text { RANKL/OPG for exp T sign > } \\
\text { cont after } 24 \mathrm{~h} \text { RANKL/OPG in } \mathrm{A}<J\end{array}$ \\
\hline \multirow[t]{3}{*}{31} & \multirow[t]{3}{*}{$\mathrm{IL}-2,6,8$} & \multirow{3}{*}{$\begin{array}{l}\text { 1-way paired } t \\
\text { Mann-Whitney } U\end{array}$} & \multirow[t]{3}{*}{ NM } & \multirow[t]{3}{*}{ NM } & IL-2-inc & \multirow{3}{*}{$\begin{array}{l}\text { IL-2 - } 7 d, 21 d \\
\text { of level IL-8 - } \\
7 d \text { of level } \\
\text { and } 7 d, 21 d \\
\text { of retr }\end{array}$} & \multirow{3}{*}{$\begin{array}{l}\text { GCF vol greater on } 7 \mathrm{~d} \text { and } \\
21 \mathrm{~d} \text { of level and retr }\end{array}$} & \multirow[t]{3}{*}{ NM } & \multirow[t]{3}{*}{ IL-8 stat sign dec on $7 \mathrm{~d}$ of level } \\
\hline & & & & & IL-6-N change & & & & \\
\hline & & & & & IL-8-dec & & & & \\
\hline 32 & RANKL, OPG & Mann-Whitney U & NM & NM & $\begin{array}{l}\text { RANKL - inc } \\
\text { OPG - dec }\end{array}$ & $24 \mathrm{~h}$ & $\begin{array}{l}\text { In vitro compres } F \text { for } 3,6,9,12 \text {, } \\
24,48 \mathrm{~h} \text {, RANKL was sign inc in } \\
\text { stress (+) grp }\end{array}$ & $\begin{array}{l}\mathrm{N} \text { sign diff in mean vol of GCF } \\
\text { at } 24 \mathrm{~h} \mathrm{~b} / \mathrm{w} \text { exp T and cont T }\end{array}$ & $\begin{array}{l}\text { Mean RANKL values after } 24 \mathrm{~h} \text { in } \\
\text { Exp }>\text { cont-mean OPG values after } \\
24 \mathrm{~h} \text { in Exp }<\text { cont }\end{array}$ \\
\hline 33 & $\begin{array}{l}\text { IL-1 } 13, \text { SP, } \\
\text { PGE2 }\end{array}$ & $\begin{array}{l}\text { Paired } t \text { multiple linear } \\
\text { regression analysis }\end{array}$ & Y & NM & $\operatorname{lnc}$ & $1 d$ & $\begin{array}{l}\text { VAS inc sign } 1 \mathrm{~h} \text { and } 24 \mathrm{~h} \text { after } \\
\text { insert of sepr and returned to bas } \\
\text { after } 7 \mathrm{~d} \text { SP and PGE2 sign higher } \\
\text { at } 1 \mathrm{~d} \text { and } 7 \mathrm{~d}\end{array}$ & NM & IL-1 $\beta$ of exp $>$ cont at $1 \mathrm{~h}, 1 \mathrm{~d}, 7 \mathrm{~d}$ \\
\hline 34 & $S P, I L-1 \beta$ & $\begin{array}{l}\text { Mann-Whitney U } \\
\text { Spearman's signed rank }\end{array}$ & NM & NM & $\operatorname{lnc}$ & NM & $\begin{array}{l}\text { Avg amt of TM was } 1.5 \pm 0.4 \mathrm{~mm} \\
\text { over } 168 \mathrm{~h}-\mathrm{N} \text { sign diff in tot prot } \\
\text { level at any of exp time periods } \\
\text { mean SP levels inc after 8, 24, } \\
\text { and } 72 \mathrm{~h} \text { in Exp }\end{array}$ & $\begin{array}{l}\mathrm{N} \text { sign diff in mean GCF vol } \\
\mathrm{b} / \mathrm{w} \text { exp and cont } \mathrm{T}\end{array}$ & $\begin{array}{l}\text { mean IL-1 } \beta \text { levels inc after 8, 24, and } \\
72 \mathrm{~h} \text { in Exp }\end{array}$ \\
\hline
\end{tabular}


Table 5 Result characteristics (Continued)

$35\|-1 \beta\|-,1 R A$

$36 \quad$ IL-8

Mann-Whitney U

NM

$\mathrm{NM} \quad \mathrm{Inc}$

Wilcoxon

$37 \quad$ IL-1ß, PGE2

Intra-grp: ANOVA

Intergrp: 1-way ANOVA

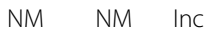

38 PGE-2, IL-6, GMCSF

$39 \quad \mathrm{LL}-1 \beta, \beta \mathrm{G}$

$40 \quad \mathrm{LL}-1 \beta$, IL-1RA, Al

41 TGF- $\beta$

$42 \mathrm{IL}-1 \beta, \mathrm{IL}-6$, TNF- $a$, EGF, $\beta 2-\mu \mathrm{G}$

19 IL-1ß, PGE

2-way analy of variancepaired $t$

NM NM Inc

Friedman, Wilcoxon test Spearman Rank Corre Analy.

IL-1 $\beta$, TNF- NO

44 RANKL, OPG

Repeated measures ANOVA

NM NM Inc

45 RANKL, HSP70,

ANOVA post hoc Fisher's LSD

46 MMP-8, IL-1 $\beta \quad$ Paired-sample $t$

47 GM-CSF, IF- $\gamma_{\text {, }}$ $\mathrm{LL}-1 \beta, \mathrm{LL}-2, \mathrm{LL}-4$ IL-5, IL-6, IL-8, IL-10 and

Paired non-parametric

Kruskall-Wallis. Spearman

Rank Sum analy.

\section{$3 d$}

dis

by lag phase with Mx C moved by 4,13 , and $26 \mathrm{kPa}$.By $52 \mathrm{kPa}$, distinct lag phase at $3 d, 14 d$

$6 \mathrm{~d}$ at tension GCF vol greater at tension and site, and 1st pressure sites at $24 \mathrm{~h}$ and $30 \mathrm{~d}$ $24 \mathrm{~h}$ at

pressure site

PGE2 inc at $24 \mathrm{~h}>\mathrm{BS}$ in $\mathrm{CF}$ and $\mathrm{NM}$

IF. PGE2 inc in CF and IF at $24 \mathrm{~h}>$

cont In CF,PGE2 > cont at $168 \mathrm{~h}$,

then fall. In IF, PGE2 remain high

for a wk.

$24 \mathrm{~h} \quad$ Median GCF vol. in J > A. In J NM

and A PGE2 inc at $24 \mathrm{~h}>\mathrm{BS}$

IL-1 $\beta-81 d \quad \beta G$ - significantly dec $14 d$ after NM

$\beta G-46 \mathrm{~d}$ prophy. Exp > cont at $46 \mathrm{~d}$

$3 \mathrm{~d} \quad C$ retr at 127 and $0.87 \mathrm{~mm} / \mathrm{m}$

for 13 and $4 \mathrm{kPa}$ of stress, resp

+ve corr of velocity and $\mathrm{Al}$

from $D>M$.

$24 \mathrm{~h}$

C retr was 1.1 at $0.1 \mathrm{~mm} / 168 \mathrm{~h} \quad \mathrm{NM}$

$24 \mathrm{~h}$

$\beta-2 M G$ inc at $24 h>B S$ or $1 h$

NM

$\beta-2 M G$ in exp at $24 \mathrm{~h}>$ ant cont
PGE inc in exp > cont at $24 \mathrm{~h}, 48 \mathrm{~h} \quad \mathrm{NM}$

PGE inc at $24 \mathrm{~h}>\mathrm{BS}, 168 \mathrm{~h}$

$\mathrm{Pl}, \mathrm{Gl}, \mathrm{PD}$ inc at $1 \mathrm{~m}>\mathrm{BS}$

$6 m>B S$

NM

PM retr $-3.73 \pm 1.08 \mathrm{~mm}$

(laser grp) and $2.71 \pm 0.9 \mathrm{~mm}$

(Cont grp) Max mean retr v(t)

btw 0 and $48 \mathrm{~h}$. Pain intensity

pk at $48 \mathrm{~h}$.

$24 \mathrm{~h} \quad$ Amylase activity in saliva inc

at $24 \mathrm{~h}$ at $\exp >$ cont

NM

$3 \mathrm{~m}$

+ve corr of GCF vol and plaque index at 0 at $(\mathrm{t})$, (comp)

Exp - TIMP 1 and 2 inc at $4 \mathrm{~h}, 7$ $d$ and $42 d, M M P-9$ inc at $4 h, 7 d$. vs avg Al in GCF from D sites (a)

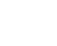

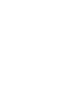

(1)

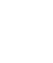

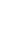


Table 5 Result characteristics (Continued)

TNFa, MMP-9,

TIMP-1 and 2

RANKL, OPG

48 RANK, OPG, OPN, TGF- $\beta$

49 MMP-3, MMP-9, MMP-13, MIP-1 MCP-1, RANTES

$50 \quad \mathrm{LL}-1 \beta, \mathrm{SP}, \mathrm{PGE} 2$

Friedman Dunn's multiple comparisons as post hoc

Friedman, Mann-Whitney $Y \quad$ NM NM

SPSS 13.0 paired $t$-test $\quad$ NM NM Inc Wilcoxon paired signed rank

$51 \quad \mathrm{IL}-1 \beta$

52 IL-2, IL-6, IL-8

53 CCL-2 (MCP1), CCL-3, CCL-5 (RANTES), IL-8 (CXCL8), IL-1a, IL-1 $\beta, \|-6$, TNF-a

54 TNF-a

Paired $t$ (intra G)1-way NM NM Inc ANOVA (interG) Dunnett's

t Tukey's

55 TNF- $a$, IL-1 $\beta$, IL-8 1-way ANOVA Paired NM NM TNF-a, IL-1 $\beta$,

student $t$ IL-8 inc in 1

to 2 d of level

Least square Regression Pearson product-moment Correl coefficient

57 RANKL, OPG, IL-1, Repeated measures IL-1RA, MMP-9 models
Compr-MMP -9 inc at $4 \mathrm{~h}$ and 7 plaque-induced inflam at 0

d. TIMP-1 inc at $4 \mathrm{~h}$ and TIMP-2 at (comp).

Exp > cont: MMP-9 inc at $0,4 \mathrm{~h}$ at compr, TIMP-2 inc at 0 at Exp, compr

\section{$\mathrm{NM}$}

MMPs inc at $1 \mathrm{~h}$, dec at $24 \mathrm{~h}$. GCF vol at $(\mathrm{comp})>(\mathrm{t})$ at $21 \mathrm{~d}$

Exp > Cont:

At $D, S P$ inc at $1 d, 7 d$

At D, PGE2 inc at $1 \mathrm{~min}, 1 \mathrm{~h}$, $1 \mathrm{~d}, 7 \mathrm{~d}$.

At $\mathrm{M}, \mathrm{PGE} 2, \mathrm{SP}$, inc at $1 \mathrm{~min}$

$1 \mathrm{~h}, 1 \mathrm{~d}, 7 \mathrm{~d}>\mathrm{BS}$

At $D, S P, P G E 2$ inc at $1 d, 7 d>B S$.

LL-1 $\beta$ at $24 h, 48$ h at exp > implant NM

IL-8 inc at $24 \mathrm{~h}, 48 \mathrm{~h}$ in MS grp NM

IL-6 inc at $3 \mathrm{~m}$ in MS grp

MOPs inc the rate of $C$ retr by 2.3-fold compd to cont and contr C - VAS sign at $24 \mathrm{~h}$ for Exp and Con

MOPs sign inc cyt and chemokine expression

NM

NM

GCF vol inc in 1 to $3 \mathrm{~d}$ of leveling NM

C retr at day 84 for 13, 26, $52 \quad \mathrm{v}(\mathrm{t})$ affected by Al in GCF, $\mathrm{kPa}$ were $4.14 \pm 0.19,6.36 \pm 1.32$, stress and IL-1 gene cluster $5.66 \pm 1.38 \mathrm{~mm}$ resp

NM

NM
NM IL-1/LL-1+

IL-1RA -
decRANKL/

RANKL +

$\mathrm{OPG}$ - inc
RANK - inc in $7 d$ in exp, compres $>$ cont OPG - cont $>$ compres site at 24 TGF- $\beta$ - inc in compres $>$ cont at $7 \mathrm{~d}$.

NM

Exp > Cont:

At M, IL-1 $\beta$ inc at $1 \mathrm{~min}, 1 \mathrm{~h}, 1 \mathrm{~d}, 7 \mathrm{~d}$. At $D, I L-1 \beta$ at $1 \mathrm{~h}, 1 \mathrm{~d}, 7 \mathrm{~d}$.

Exp > bas: At M, IL-1 $\beta$ inc at 1 min,

$1 \mathrm{~h}, 1 \mathrm{~d}, 7 \mathrm{~d}$. At $\mathrm{D}, \mathrm{LL}-1 \beta$ inc

at $1 \mathrm{~h}, 1 \mathrm{~d}, 7 \mathrm{~d}$.

IL-1 $\beta$ inc at $24,48 h>$ bas IL-1 $\beta$ dec after $24 \mathrm{~h}$, at $48 \mathrm{~h}, 168 \mathrm{~h}, 14 \mathrm{~d}, 21 \mathrm{~d}$

IL-2 inc at $24 h>$ bas

IL-8 inc in Exp at $1 \mathrm{~h}, 24 \mathrm{~h}, 48 \mathrm{~h}$

All cyt and chemo inc in both Cont and Exp at $24 \mathrm{~h} \mathrm{IL-1}$ inc also at $28 \mathrm{~d}$

TNF-a inc at $24 \mathrm{~h}$

TNF-a, IL-1 $\beta, ~ I L-8$ inc in 1 to $2 \mathrm{~d}$ of level

Faster $v(\mathrm{t})$ seen in $26 \mathrm{kPa}$, higher GCF and allele 1 homozygosity

IL-1RA in adults exp > cont at 3 wk. Dec in ratio of (IL-1/[LL-1 + IL-1RA]). Inc in ratio of RANKL to OPG (RANKL/[RANKL + OPG]). RANKL to OPG inc at 6 wk in ado, at $3 w k$ in $\mathrm{A}$, OPG in ado at $\operatorname{Exp}<$ cont at 6 wk 
Table 5 Result characteristics (Continued)

$58 \quad \mathrm{LL}-2, \mathrm{LL}-4, \mathrm{LL}-6, \quad$ Stepwise regression NM

$\mathrm{IL}-8, \mathrm{IL}-10, \mathrm{GM}-\mathrm{CSF}$

IFN- - , TNF-a,

59 OPG, RANKL

2-way ANOVA.A

Bonferroni if

NM NM

NM

NM

NM

L-6 levels at bas predictive of

No sign change

GCF flows after 1 y of ortho t/t NM NM $\begin{aligned} & \text { OPG inc in } M, \\ & \text { dec in D at } 1 \mathrm{~h}\end{aligned}$

NM

NM

OPG inc on $\mathrm{M}$, dec in $\mathrm{D}$ at $1 \mathrm{~h}$

Ref No. reference number, Stats statistically, analy analysis, appld applied, Confd confounders, reg regulation, $P k$ peak, Sec secondary, outcm outcome, correl correlation, sign significant, $Y$ yes, $N$ no, NM not mentioned, inc increase, dec decrease, fluct fluctuated, $h$ hour, $m$ month, tot total, prot protein, conc concentration, $m g$ milligram, $m /$ milliliter, $g$ gram, $>$ greater than, $<$ less than, VAS visual analog scale, $C$ canine, mov movement, $b / w$ between, CF continuous force, IF interrupted force, and and, $F$ force, Assoc associated, gen genetic, GCF gingival crevicular fluid, compd compared, bas baseline, IL interleukin, $\beta G$ beta glucoronidase, TNF- $a$ tumor necrosis factor alpha, SD short duration, $L D$ long duration, Diff difference, vol volume, retr retraction, correl correlation, inflam inflammation, Avg average, cyt cytokine, chemo chemokine, knwn known, MOPs microosteoperforations, PI plaque index, BOP bleeding on probing, exp experimental, cont control, Exp experimental tooth, ant antagonistic, Avg average, Mx maxilla, contr contralateral, differen differentiation, sepr separator, grp group, compres compression, $k P a$ kilopascal, max maximum, grw growth, $T$ tooth, Oc osteoclast, $R D G$ rapid canine distalization group, $H G$ hybrid reactor group. $v(t)$ velocity of tooth movement, $M$ mesial, $D$ distal, level leveling, retr retraction.

CSFs colony-stimulating factors, IFNs interferons, MCSF macrophage colony-stimulating factor, SP substance P, IL-1 $\beta$ interleukin-1 beta, TNF- $a$ tumor necrosis factor-alpha, TGF $\beta$ transforming growth factor-beta, OPG osteoprotegerin, OPN osteopontin, RANKL receptor activator of nuclear factor kappa-B ligand, RANK receptor activator of nuclear factor kappa-B, GM-CSF granulocyte-macrophage colony-stimulating factor, $\beta G$ beta glucuronidase, PGE2 prostaglandin E2, IL-1RA interleukin receptor antagonist, MCP monocyte chemoattractant protein, MMP matrix metalloproteinases, MIP macrophage inflammatory protein, TIMP tissue inhibitor of metalloproeintases, HSP heat shock proteins, NO nitric oxide, Al activity index. 
time for GCF collection. The room temperature conditions were considered in six $(\mathrm{N}=6)$ studies and humidity in five $(\mathrm{N}=5)$ studies.

The sites for GCF sample collection were either mesial $(\mathrm{N}=5)$ or distal $(\mathrm{N}=21)$ or both mesial and distal $(\mathrm{N}=12)$. GCF collection was done by periopaper $(\mathrm{N}=33)$ studies, filter paper $(\mathrm{N}=3)$, or filter membrane $(\mathrm{N}=2)$. One study $(\mathrm{N}=1)$ did not mention the technique by which GCF was collected.

Depth of insertion of paper for GCF collection was mentioned in 27 studies with the most common practice being a 1-mm depth $(\mathrm{N}=26)$. One study used 2-mm depth of insertion. Duration of GCF collection was specified to be $30 \mathrm{~s}$ in most studies $(\mathrm{N}=29)$ followed by 60 $\mathrm{s}(\mathrm{N}=4), 3 \mathrm{~min}(\mathrm{~N}=1), 10 \mathrm{~s}(\mathrm{~N}=1)$, and $20 \mathrm{~s}(\mathrm{~N}=1)$. Repeated measurements were considered in 19 studies $(\mathrm{N}=19)$ with collection repeated once in $\mathrm{N}=11$ studies, twice in $\mathrm{N}=5$ studies, and 3 times in $\mathrm{N}=3$ studies. The interval was $60 \mathrm{~s}(\mathrm{~N}=14), 90 \mathrm{~s}(\mathrm{~N}=1)$, and $120 \mathrm{~s}(\mathrm{~N}=1)$.

The samples were stored at $-20^{\circ} \mathrm{C}(\mathrm{N}=7),-30^{\circ} \mathrm{C}$ $(\mathrm{N}=6),-70^{\circ} \mathrm{C}(\mathrm{N}=10)$, and $-80^{\circ} \mathrm{C}(\mathrm{N}=9)$. The GCF from periopaper was retrieved by Periotron $(\mathrm{PT}) 8000(\mathrm{~N}=$ 18), PT6000 ( $=5)$, PT $(\mathrm{N}=2)$, or electronic scale $(\mathrm{N}=3)$ and was not mentioned in some studies $(\mathrm{N}=11)$. Mediators were analyzed by ELISA $(\mathrm{N}=27)$, immunoassay (IA) $(\mathrm{N}=$ 8), Luminex multianalyte technology (LMAT) $(\mathrm{N}=1)$, BioPlex human cytokine assay (BHCA) $(\mathrm{N}=1)$, custom protein array $(\mathrm{CPA})(\mathrm{N}=1)$, or Quantibody Ar kit (QAK) $(\mathrm{N}=1)$. Protein concentration in GCF was measured in $\mathrm{pg} / \mu \mathrm{l}(\mathrm{N}=$ 12), pg $(\mathrm{N}=5), \mathrm{pg} / \mathrm{mg}(\mathrm{N}=1), \mathrm{pg} / \mathrm{ml}(\mathrm{N}=5), \mathrm{pg} / \mathrm{mg}(\mathrm{N}=$ 1), $\mathrm{pg} / \mu \mathrm{g}(\mathrm{N}=30), \mathrm{pg} / 30 \mathrm{~s}(\mathrm{~N}=2), \mathrm{pg} / 20 \mathrm{~s}(\mathrm{~N}=1)$, and $\mathrm{pg} /$ site $(\mathrm{N}=1)$ and was not mentioned in $\mathrm{N}=3$ studies.

The total number of studies included in this review are 39. However in the result section, some of the variables showing number of studies may be more than 39 , as few parameters have been divided into subgroups that have been considered as a separate variable.

\section{Mediator levels in GCF}

Interleukins Twenty-one studies on IL- $1 \beta$ were evaluated. Of these, ten studies $(\mathrm{N}=10)$ reported that the peak levels of IL-1 $\beta$ were attained at $24 \mathrm{~h}[19,22,27,33,37,42,50,51,53,54]$. The peak levels in other studies have also been reported at 4 h [47], 3 days [35,40], 7 days, 21 days [29], 2 months [22], around 3 months [39,46], and 6 months [43]. One study mentioned peak for different teeth at different observation points [26] that resulted in peak for molars at 67 days, for premolars at 32 days and for central incisors at 39 days. Another study did not mention peak but fluctuation in IL- $1 \beta$ levels on application of different stresses of teeth that were correlated with velocity [24].

One study mentioned IL-1 $\beta$ levels on application of 150 -g force to be twice that on application of 50 -g force at $24 \mathrm{~h}$ and 2 months [22]. Studies have shown decrease in levels of
IL-1 $\beta$, 14 days after prophylaxis, followed by an increase upon activation of orthodontic appliance $(\mathrm{N}=2)$ [26,39]. Forces of short duration show an increase in IL-1 $\beta$ at 7 and 21 days [29]. Levels of IL-1 $\beta$ in experiment teeth were shown to be greater than control teeth at $1 \mathrm{~h}[19,33], 4 \mathrm{~h}[47], 8 \mathrm{~h}$ [34], $24 \mathrm{~h}$ [19,33,34,42],72 $\mathrm{h}$ [34], 7 days [33], 25 days [26,39], 32 days [26,39], 33 days [39], 39 days [26,39], 42 days [47], 46 days [26,39], 67 days [26,39], and 81 days [26,39].

A comparison of continuous and interrupted force was evident in one study [37] where it was shown that IL-1 $\beta$ levels in continuous force is greater than baseline at $24 \mathrm{~h}$ while in interrupted force, levels were greatest at first reactivation.

Difference in IL-1 $\beta$ levels according to site specification was mentioned in one study [40] where levels at distal site of tooth retraction were greater than mesial site at both 4 and $13 \mathrm{kPa}$ of force application. Placement of elastic separators in molars led to an increase in levels at $1 \mathrm{~min}, 1 \mathrm{~h}$, $24 \mathrm{~h}$, and 7 days [50] at mesial site while at distal site, increase was seen at $1 \mathrm{~h}, 24 \mathrm{~h}$, and 7 days [50].

An upregulation in IL-1 $\beta$ levels from baseline levels was evident at $1 \mathrm{~min}$ [50], $1 \mathrm{~h}$ [50], $24 \mathrm{~h}$ [19,37,42,51,54], $48 \mathrm{~h}$ [51], 7 days [50], 3 months [46], and 6 months [43] and downregulation was seen in $48 \mathrm{~h}, 168 \mathrm{~h}, 14$ days, and 21 days [51].

For IL-6, the levels were found to increase at $24 \mathrm{~h}$ $[38,53]$ when continuous forces were applied for retraction or tipping.

IL-8 also increased on application of continuous force for retraction at $1 \mathrm{~h}[36,52]$ both on tension and pressure sites [36] and also at $4 \mathrm{~h}$ [47], 24 h, and $48 \mathrm{~h}$ [52]. Placement of separators led to an increase in levels at $24 \mathrm{~h}$ [27]. Fall in levels was observed at 7 days of leveling and an increase was seen at 7 days, 21 days of retraction [31].

TNF- $\boldsymbol{\alpha}$ Application of interrupted force witnessed an increase in levels at $1 \mathrm{~h}[28]$ and $24 \mathrm{~h}[27,42]$ while continuous force application led to increase in levels at $24 \mathrm{~h}$ $[53,54]$ or $4 \mathrm{~h}$ to 42 days [47]. There was a decrease in levels in 1 week on continuous force retraction by hybrid retractor (HG) [28]. TNF- $\alpha$ levels also increased at 1 day [54], at 3 months [29] of leveling, and at 6 months, just before retraction [29]. A comparison of continuous force application by hybrid retractor (HG) by HG to interrupted force by rapid canine distalizer (RDG) showed higher values at $1 \mathrm{~h}$ in RDG group compared to HG [28].

\section{Levels of receptor and their antagonists in GCF RANKL}

RANKL showed an increase in levels at $24 \mathrm{~h}[30,32,45]$ greater than control as well as baseline with specific mention of levels in juveniles and adults [30,32]. Two studies $(\mathrm{N}=2)$ mentioned increase in levels greater than baseline, one at $48 \mathrm{~h}$ [44] and other at 42 days [47] at 
24 h greater than control [45], at $48 \mathrm{~h}$ [44], 42 days [47] greater than baseline. Correlation with age was established with levels in adults being less than juveniles [30,32], an increase in RANKL/OPG ratio in 6 weeks in adolescents [57].

\section{IL-1RA}

Lower value IL-1RA was shown to be associated with higher velocity of tooth movement $(\mathrm{Vt})$ [24]. It was also a determinant of activity index (AI) that is ratio of concentrations of IL-1 $\beta$ and IL-1RA in GCF, known to correlate with $\mathrm{Vt}[24,35,40,56]$. One study mentioned distinction of site where levels at distal site of retraction were greater than mesial and control on application of 4 and $13 \mathrm{kPa}$ force [40]. A comparison between levels in adults and adolescents revealed a decrease in ratio of IL1/(IL1 + IL-1RA) in 3 weeks [57].

\section{OPG}

The levels were decreased in experimental teeth at $1 \mathrm{~h}$ [25], $24 \mathrm{~h}$ [25,30,48], $168 \mathrm{~h}$ [25], 1 month [25], and 3 months [25] than baseline levels. A distinction of age-specified levels of OPG as well as ratio of RANKL/OPG was found to be lower in adults than in juveniles [30]. The levels of OPG in experimental teeth were found to be lower in adolescents in 6 weeks compared to control teeth [57]. Its values were less in adolescents in 6 weeks [57]. Site specification determined levels to increase on tension site (mesial) and to decrease on compression site (distal) [59].

Chemokines [CCL-2 (MCP1), CCL-3, CCL-5 (RANTES), IL-8 (CXCL8)] showed an increase in both experimental and control teeth at $24 \mathrm{~h}$ of force application [53]. Levels of IL-8 were increased when force was applied for a short duration in separator placement [27], or longer duration in initial alignment [27], 1 to 2 days of leveling [54], increase from $4 \mathrm{~h}$ to 42 days [47], at $1 \mathrm{~h}, 24 \mathrm{~h}$, and $48 \mathrm{~h}$ [52] when continuous forces were applied for retraction. Levels also showed a decrease in 7 days of leveling [31]. A difference in levels was observed with distinction of site, with levels increased at both mesial and distal sites at $1 \mathrm{~h}$ of force application, between $24 \mathrm{~h}$ and 6 days at tension site and a statistically significant increase at tension site greater than compression site [36]. Two studies $(\mathrm{N}=2)$ on chemokines did not reveal any significant findings $[49,58]$.

\section{Discussion}

This systematic review was primarily aimed to conjure substantial evidence regarding the cytokine, chemokine, receptor and their antagonist (RANK, RANKL, OPG) levels in GCF consequent to application of orthodontic force. The literature revealed heterogeneity in study designs pertaining to participant characteristics, force application, levels of force, GCF collection methods and collection protocol, storage, and oral hygiene maintenance regimen. To draw logical conclusions each of the variables was tabulated and analyzed separately. Associations of change in levels of mediators were established with mechanics of applied orthodontic force, amount of force, force reactivations, differentiation in levels between tension and compression sites, age groups (juveniles and adults, growers and non-growers), and velocity of tooth movement $(\mathrm{Vt})$.

The altering levels, rise, and fall of the mediators in GCF are suggestive of underlying intricate biological remodeling processes in bone and periodontal tissues that eventually leads to OTM. The forces employed for OTM or midpalatal expansion led to an initial increase in levels of bone-resorptive mediators as well as associated receptors namely IL- $1 \beta$, IL- 8 , RANKL, and TNF- $\alpha$ as early as $1 \mathrm{~min}$ [50] or $1 \mathrm{~h} \mathrm{[28]} \mathrm{and} \mathrm{attained} \mathrm{peak} \mathrm{in} 24 \mathrm{~h}$ $[19,22,27,30-33,37,42,45,50,51,53,54]$. These mediators slowly decrease to baseline in subsequent observation points at $48 \mathrm{~h}, 168 \mathrm{~h}, 14$ days, and 21 days [28,31,51]. On the contrary, bone-forming mediators like OPG show an immediate decrease in levels on application of orthodontic forces at $1 \mathrm{~h}$ on distal site of retraction [25,59], at $24 \mathrm{~h}$ $[25,30,32,48]$. The role of RANK, RANKL, and OPG system in governing osteoclastogenesis has also been corroborated in animal studies $[61,62]$ and in vitro studies [63-66] on periodontal ligament cells. When compressive orthodontic force is applied, upregulation of RANKL occurs which leads to stimulation of PGE2 pathway and finally, osteoclastic activity is initiated which results in bone resorption $[65,66]$. OPG, a RANKL decoy receptor generated by osteoblastic cells and cells of the periodontal ligament, binds to RANKL and inhibits RANK/RANKL interaction that is the mainstay of osteoclastogenesis [67].

Besides these receptors, other factors that are directly or indirectly responsible for differentiation, survival, and activity of osteoclasts are cytokines (IL-1 $\beta$, TNF $\alpha$, IL-6) and chemokines (CCL2, CCL3, CCL5, CCL7, CCL9, IL-8) $[68,69]$. The literature search in the present review found an increase in levels of these mediators in GCF on orthodontic force application. Evidence proves that mechanical stress induces acute inflammatory changes that alter the microvascular environment, with studies supporting local release of mediators IL-1 $\beta$, TNF- $\alpha$, and expression of chemokines that ultimately promotes leukocyte adhesion and migration [70]. IL-1 $\beta(\mathrm{N}=21)$ and TNF- $\alpha(\mathrm{N}=10)$ are the most researched cytokines, supporting their role in the inflammatory changes associated with orthodontic tooth movement (OTM). Variation in mediator levels with type of force and force reactivations has also been evaluated to study their clinical implications with IL-1 $\beta$, PGE2, or TNF- $\alpha$ levels showing an initial increase, both in continuous and interrupted force [28,37]. However, timely reactivations in interrupted force led to an upregulation of these mediators, indicative of greater inflammation than 
on continuous force application [28,37]. This finding is in accordance with other studies that support association of light continuous forces for OTM with minimal inflammation, root resorption, and hyalinization of the periodontal ligament [71-73]. More recent techniques for accelerated orthodontics like micro-osteoperforations have also conducted studies at cellular level that led to increase in GCF levels of cytokines ( IL-1 $\alpha$, IL-1 $1 \beta$, IL-6, TNF- $\alpha$ ) and chemokines at $24 \mathrm{~h}$, giving evidence of underlying inflammatory process associated with inducing perforations in cortical bone [53]. An animal study further supports the release of proinflammatory cytokines with micro-osteoperforations, known to recruit osteoclast precursors and hence increase OTM by influencing the bone remodeling process [74].

Results of the review showed that compression side witnessed a decrease in bone-formative OPG by $24 \mathrm{~h}$ [48] and increase in bone-resorptive RANK and TGF- $\beta 1$ after 7 days $[32,48]$. Other mediators showing temporal variation on compression side were IL- $1 \beta$ that increased as early as $1 \mathrm{~min}$ [50] or after $4 \mathrm{~h}$ [47], RANKL after 42 days [47] or after $24 \mathrm{~h}$ both in juveniles and adults [30], and IL-8 after $4 \mathrm{~h}$ [47] or after 10 days [36]. In contrast, the tension site showed an appreciable increase in TNF- $\alpha$ [47] and other bone-resorbing mediators like IL$1 \beta$, PGE2, and IL-8. But the rise occurred earlier than compression and at all observation points with levels higher than at the compression site [36,47]. This difference is hard to understand as the concept of compression on one side and tension on the other side of the tooth undergoing movement is hypothetical. It has been logically contradicted since the anatomical shape and surface morphology of the tooth root cannot be considered confined to definite geometry. Thus, forces when applied lead to biological response in whole of the periodontal apparatus that cannot be differentiated for release of inflammatory mediators in GCF which is a freely circulating fluid in gingival sulcus. Therefore, mediator levels in GCF collected from mesial or distal sites of the tooth may not be indicative solely of compression or tension zone activity.

Synopsis of the studies included in the review also revealed that age and growth status were factors influencing the level of cytokines in GCF that is shown to have an effect on the rate and amount of tooth movement. Mediator levels were seen to vary with growth status of individuals as evaluated in adolescents and adults [57] or compared in juveniles and adults [30,38]. In one study, different mediators were found to increase in different age groups with IL-6, GM-CSF increasing only in juveniles while PGE2 increasing both in juveniles and adults [38]. In addition, activity index (AI) that is the ratio of IL-1 $\beta /$ IL-1RA in GCF, was found to influence velocity $(\mathrm{Vt})$ of OTM both in growers and non-growers [35].
Mean Vt of growers was $0.050 \mathrm{~mm} /$ day and of nongrowers was $0.024 \mathrm{~mm} /$ day for the same amount of applied stress that was correlated with higher levels of IL-1 $\beta$ and an increased AI in growers [35]. A greater Vt and amount of OTM in juveniles as compared to adults could be explained on the basis of a higher RANKL/OPG ratio in GCF in juveniles [30]. Other studies also support the variation in Vt according to varying mediator levels and AI in GCF $[24,35,40,56]$. It was found that association between AI and $\mathrm{Vt}$ was stronger from distal than from mesial of retracted teeth, thus emphasizing greater values of IL$1 \beta$ on the distal [40]. Thus, evidence from this literature review emphasizes the role of RANKL/OPG ratio in OTM in either of the age groups owing to its significance in osteoclastogenesis and bone resorption that ultimately alters the amount and velocity of OTM.

Secondary outcome of this review was the association of intensity of pain with different force levels. The level of IL-1 $\beta$ was seen to increase at 1 day [50] that correlated with increased pain intensity and subsequently, there was a decrease seen in 7 days. Another study suggested pain was less with 50 -g force as compared to 150 -g force that was correlated with greater levels of IL$1 \beta$ with application of 150 -g force [22]. It can be concluded that $150 \mathrm{~g}$ force is marked by higher levels of IL-1 $\beta$ in GCF compared to $50-\mathrm{g}$ force and high pain intensity.

\section{Conclusions}

This systematic review is focused on association of cytokine and receptor levels or activity index in GCF with velocity of tooth movement, nature of force applied, pain intensity, and growth status/age of the subjects, leading to following conclusions:

1. Application of orthodontic forces causes an immediate release of inflammatory bone-resorptive mediators (IL-1 $\beta$, TNF- $\alpha$ ) in $1 \mathrm{~h}$ that reach peak in $24 \mathrm{~h}$, thus supporting the role of inflammation in initial OTM.

2. Bone-forming mediators like OPG witnessed a fall in levels immediately after orthodontic force application indicating bone resorption to be the key process in initiating tooth movement.

3. The levels of cytokines decrease after attaining peak values, mostly at $24 \mathrm{~h}$ in continuous forces but repeated activations in interrupted force upregulate their secretion.

4. A rise in GCF levels of IL-1 $\beta$ with higher force levels (150 vs $50 \mathrm{~g}$ ) has been linked to increased pain intensity during OTM.

5. Juveniles exhibiting greater RANKL/OPG ratio and activity index (AI) (IL-1 $\beta / I L-1 R A)$ in GCF displayed faster rate of OTM than adults or non-growers. 
6. Increased velocity of tooth movement $(\mathrm{Vt})$ has been correlated with a greater activity index (AI) in GCF.

The literature search and critical review have also provided a lead to lacunae of the research in this field. There is lack of uniformity of study design with respect to sample size, age, sex ratio, observation intervals, duration of observations, mechanism employed to initiate OTM and ethnic/nutritional barriers. These are potential confounders which can influence the outcome [75]. A major drawback identified in the current review was the lack of consideration of sex on mediator levels that are known to be sensitive to estrous cycle. Animal studies have evaluated correlation between ovarian activity and PGE2, IL-1 $\beta$ levels in GCF of female cats during OTM. Results revealed that mediator levels of estrous groups were lower than anestrous and ovariectomized groups on 6 and 12 days, indicating that ovarian activity can affect OTM [76]. The threshold levels of the inflammatory mediators for initiation for OTM also remain unexplored. Besides GCF, peri-implant fluid may also be a potential medium to study these markers noninvasively in future studies [77]. Search of pain killers having least effect on bone-resorbing mediator levels as a drug of choice may be a potential area of future research [78]. Research related to the role of mediators in external apical root resorption (EARR) and relapse has also received little attention and are important research areas requiring further exploration.

\section{Additional files}

Additional file 1: Annexure 1. Search Strategy.

Additional file 2: Annexure 2. Quality assessment instrument.

\section{Competing interests}

The authors declare that they have no competing interests.

\section{Authors' contributions}

PK and NM retrieved the articles for the study and compiled them into manuscript form. OPK gave the concept of this descriptive review, crosschecked the studies, and was actively involved in writing and reviewing the manuscript. RM and SK formulated the preparation of the manuscript. All authors read and approved the final manuscript.

\section{Acknowledgement}

The authors are grateful to Dr Sushma Chaurasia who provided valuable inputs for the study.

\section{Financial competing interests}

In the past five years, have you received reimbursements, fees, funding, or salary from an organization that may in any way gain or lose financially from the publication of this manuscript, either now or in the future?

Is such an organization financing this manuscript (including the article-processing charge)? If so, please specify.

This project did not receive any grant from any institution. No organization is financing this manuscript or the article-processing charge.

Do you hold any stocks or shares in an organization that may in any way gain or lose financially from the publication of this manuscript, either now or in the future? If so, please specify.
The authors do not hold any stocks and shares in any organization that may in any way gain or lose financially from the publication of this manuscript, either now or in the future.

Do you hold or are you currently applying for any patents relating to the content of the manuscript? Have you received reimbursements, fees, funding, or salary from an organization that holds or has applied for patents relating to the content of the manuscript? If so, please specify.

The authors have not applied for any patents relating to the content of the manuscript.

Do you have any other financial competing interests? If so, please specify. The authors have no other financial competing interests.

\section{Non-financial competing interests}

Are there any non-financial competing interests (political, personal, religious, ideological, academic, intellectual, commercial, or any other) to declare in relation to this manuscript? If so, please specify.

There are no non-financial competing interests in relation to this manuscript.

\section{Author details}

${ }^{1}$ Department of Orthodontics, Faculty of Dentistry, Jamia Millia Islamia, New Delhi 110025, India. ${ }^{2}$ Division of Orthodontics and Dentofacial Deformities, Centre for Dental Education and Research, All India Institute of Medical Sciences, New Delhi 110029, India. ${ }^{3}$ Department of Orthodontics and Pediatric Dentistry, Graduate Orthodontics Program Director, The University of Michigan School of Dentistry, 1011 North University Avenue, Ann Arbor, MI 48109-1078, USA.

Received: 10 September 2014 Accepted: 11 November 2014 Published online: 09 December 2014

\section{References}

1. Gianni E. Genetics and dynamical modulators in orthodontics (Italian). WFO Gazzette. 2013; 18(2):4.

2. Meikle MC. The tissue, cellular, and molecular regulation of orthodontic tooth movement: 100 years after Carl Sandstedt. Eur J Orthod. 2006; 28(3):221-40.

3. Masella RS, Meister M. Current concepts in the biology of orthodontic tooth movement. Am J Orthod Dentofacial Orthop. 2006; 129(4):458-68.

4. Uitto VJ. Gingival crevice fluid - an introduction. Periodontol 2000. 2003; 31:9-11

5. Meager A. Cytokine regulation of cellular adhesion molecule expression in inflammation. Cytokine Growth Factor Rev. 1999; 10(1):27-39.

6. Saito S, Ngan P, Saito M, Kim K, Lanese R, Shanfeld J, Davidovitch Z. Effects of cytokines on prostaglandin E and CAMP levels in human periodontal ligament fibroblasts in vitro. Arch Oral Biol. 1990; 35(5):387-95.

7. Mundy GR. Cytokines and local factors which affect osteoclast function Int J Cell Cloning. 1992; 10(4):215-22.

8. Kimoto S, Matsuzawa M, Matsubara S, Komatsu T, Uchimura N, Kawase T, Saito S. Cytokine secretion of periodontal ligament fibroblasts derived from human deciduous teeth: effect of mechanical stress on the secretion of transforming growth factor-beta 1 and macrophage colony stimulating factor. J Periodontal Res. 1999; 34(5):235-43.

9. Ozaki K, Leonard WJ. Cytokine and cytokine receptor pleiotropy and redundancy. J Biol Chem. 2002; 277:29355-58.

10. Yue Y, Liu Q, Xu C, Ty Loo W, Wang M, Wen G, Nb Cheung M, Bai LJ, Dou YD, Wc Chow L, Hao L, Tian Y, Li JL, Ys Yip A, Ly NE. Comparative evaluation of cytokines in gingival crevicular fluid and saliva of patients with aggressive periodontitis. Int J Biol Markers. 2013; 28(1):108-12.

11. Roberts WE, Huja S, Roberts JA. Bone modeling: biomechanics, molecular mechanisms, and clinical perspectives. Sem Orthod. 2004; 10:123-61.

12. Alhashimi N, Frithiof L, Brudvik P, Bakhiet M. Orthodontic tooth movement and de novo synthesis of proinflammatory cytokines. Am J Orthod Dentofacial Orthop. 2001; 119:307-12.

13. Gowen M, Wood DD, Ihrie EJ, McGuire MK, Russell RG. An interleukin 1 like factor stimulates bone resorption in vitro. Nature. 1983; 306:378-80.

14. Boyle WJ, Simonet WS, Lacey DL. Osteoclast differentiation and activation. Nature. 2003; 423:337-41.

15. Teitelbaum SL. Bone resorption by osteoclasts. Science. 2000; 289:1504-08.

16. Karsenty G. The complexities of skeletal biology. Nature. 2003; 423:316-18.

17. Theoleyre S, Wittrant Y, Tat SK, Fortun Y, Redini F, Heymann D. The molecular triad OPG/RANK/RANKL: involvement in the orchestration of 
pathophysiological bone remodeling. Cytokine Growth Factor Rev. 2004; 15(6):457-75.

18. Davidovitch Z, Nicolay OF, Ngan PW, Shanfeld JL. Neurotransmitters, cytokines and the control of alveolar bone remodeling in orthodontics. Dent Clin North Am. 1988; 32:411-35.

19. Grieve W, Johnson GK, Moore RN, Reinhardt RA, Dubois LM. PGE and IL-1 levels in gingival crevicular fluid during human orthodontic tooth movement. Am J Orthod Dentofacial Orthop. 1994; 105:369-74.

20. Kuijpers MAR, Chiu Y-T, Nada RM, Carels CEL, Fudalej PS. Three-dimensional imaging methods for quantitative analysis of facial soft tissues and skeletal morphology in patients with orofacial clefts: a systematic review. PLoS One. 2014; 9(4):e93442. doi:10.1371/journal.pone.0093442.

21. Whiting P, Rutjes AW, Reitsma JB, Bossuyt PM, Kleijnen J. The development of QUADAS: a tool for the quality assessment of studies of diagnostic accuracy included in systematic reviews. BMC Med Res Methodol. 2003; 10(3):25.

22. Luppanapornlarp S, Kajii TS, Surarit R, lida J. Interleukin-1 beta levels, pain intensity, and tooth movement using two different magnitudes of continuous orthodontic force. Eur J Orthod. 2010; 32(5):596-601.

23. Dilsiz A, Kiliç N, Aydin T, Ates FN, Zihni M, Bulut C. Leptin levels in gingival crevicular fluid during orthodontic tooth movement. Angle Orthod. 2010 80(3):504-08.

24. Iwasaki LR, Chandler JR, Marx DB, Pandey JP, Nickel JC. IL-1 gene polymorphisms, secretion in gingival crevicular fluid, and speed of human orthodontic tooth movement. Orthod Craniofac Res. 2009; 12(2):129-40.

25. Toygar HU, Kircelli BH, Bulut S, Sezgin N, Tasdelen B. Osteoprotegerin in gingival crevicular fluid under long-term continuous orthodontic force application. Angle Orthod. 2008; 78(6):988-93.

26. Tzannetou S, Efstratiadis S, Nicolay O, Grbic J, Lamster I. Comparison of levels of inflammatory mediators IL-1beta and beta $\mathrm{G}$ in gingival crevicular fluid from molars, premolars, and incisors during rapid palatal expansion. Am J Orthod Dentofacial Orthop. 2008; 133(5):699-707.

27. Ren Y, Hazemeijer H, De Haan B, Qu N, De Vos P. Cytokine profiles in crevicular fluid during orthodontic tooth movement of short and long durations. J Periodontol. 2007; 78(3):453-58.

28. Karacay S, Saygun I, Bengi AO, Serdar M. Tumor necrosis factor-alpha levels during two different canine distalization techniques. Angle Orthod. 2007; 77(1):142-47.

29. Başaran G, Ozer T, Kaya FA, Kaplan A, Hamamci O. Interleukine-1 beta and tumor necrosis factor-alpha levels in the human gingival sulcus during orthodontic treatment. Angle Orthod. 2006; 76(5):830-36.

30. Kawasaki K, Takahashi T, Yamaguchi M, Kasai K. Effects of aging on RANKL and OPG levels in gingival crevicular fluid during orthodontic tooth movement. Orthod Craniofac Res. 2006; 9(3):137-42.

31. Başaran G, Ozer T, Kaya FA, Hamamci O. Interleukins 2, 6, and 8 levels in human gingival sulcus during orthodontic treatment. Am J Orthod Dentofacial Orthop. 2006; 130(1):7.e1-6.

32. Nishijima Y, Yamaguchi M, Kojima T, Aihara N, Nakajima R, Kasai K. Levels of RANKL and OPG in gingival crevicular fluid during orthodontic tooth movement and effect of compression force on releases from periodonta ligament cells in vitro. Orthod Craniofac Res. 2006; 9(2):63-70.

33. Giannopoulou C, Dudic A, Kiliaridis S. Pain discomfort and crevicular fluid changes induced by orthodontic elastic separators in children. J Pain. 2006; 7(5):367-76.

34. Yamaguchi M, Yoshii M, Kasai K. Relationship between substance $P$ and interleukin-1 beta in gingival crevicular fluid during orthodontic tooth movement in adults. Eur J Orthod. 2006; 28(3):241-46.

35. Iwasaki LR, Crouch LD, Tutor A, Gibson S, Hukmani N, Marx DB, Nickel JC. Tooth movement and cytokines in gingival crevicular fluid and whole blood in growing and adult subjects. Am J Orthod Dentofacial Orthop. 2005; 128(4):483-91.

36. Tuncer BB, Ozmeriç N, Tuncer C, Teoman I, Cakilci B, Yücel A, Alpar R, Baloş K. Levels of interleukin-8 during tooth movement. Angle Orthod. 2005; 75(4):631-36.

37. Lee KJ, Park YC, Yu HS, Choi SH, Yoo YJ. Effects of continuous and interrupted orthodontic force on interleukin-1 beta and prostaglandin E2 production in gingival crevicular fluid. Am J Orthod Dentofacial Orthop. 2004; 125(2):168-77.

38. Ren Y, Maltha JC, Van't Hof MA, Von Den Hoff JW, Kuijpers-Jagtman AM, Zhang D. Cytokine levels in crevicular fluid are less responsive to orthodontic force in adults than in juveniles. J Clin Periodontol. 2002; 29(8):757-62.

39. Tzannetou S, Efstratiadis S, Nicolay O, Grbic J, Lamster I. Interleukin-1b and b-glucuronidase in gingival crevicular fluid from molars during rapid palatal expansion. Am J Orthod Dentofacial Orthop. 1998; 114:686-96.

40. Iwasaki LR, Haack JE, Nickel JC, Reinhardt RA, Petro TM. Human interleukin-1 beta and interleukin-1 receptor antagonist secretion and velocity of tooth movement. Arch Oral Biol. 2001; 46(2):185-89.

41. Uematsu S, Mogi M, Deguchi T. Increase of transforming growth factor-beta 1 in gingival crevicular fluid during human orthodontic tooth movement. Arch Oral Biol. 1996; 41(11):1091-95.

42. Uematsu S, Mogi M, Deguchi T. Interleukin (IL)-1 beta, IL-6, tumor necrosis factor-alpha, epidermal growth factor, and beta 2-microglobulin levels are elevated in gingival crevicular fluid during human orthodontic tooth movement. J Dent Res. 1996; 75(1):562-67.

43. Atuğ Özcan SS, Ceylan I, Ozcan E, Kurt N, Dağsuyu IM, Canakçi CF. Evaluation of oxidative stress biomarkers in patients with fixed orthodontic appliances. Dis Markers. 2014; 2014:597892. doi:10.1155/2014/597892. Epub 2014 Apr 10

44. Domínguez A, Gómez C, Palma JC. Effects of low-level laser therapy on orthodontics: rate of tooth movement, pain, and release of RANKL and OPG in GCF. Lasers Med Sci. 2013 Dec 18. doi:10.1007/s10103-013-1508

45. Kuroki H, Miyagawa Y, Shimomura-Kuroki J, Endo T, Shimomura H. Identification of marker proteins by orthodontic treatment: relationship of RANKL in the gingival crevicular fluid and of amylase in whole saliva with orthodontic treatment. Odontology. 2013 Jul 18. doi:10.1007/s10266-013-0121-6

46. Ribagin LS, Rashkova MR. Matrix metalloproteinase-8 and interleukin-1 beta in gingival fluid of children in the first three months of orthodontic treatment with fixed appliances. Folia Med (Plovdiv). 2012; 54(3):50-6

47. Grant M, Wilson J, Rock P, Chapple I. Induction of cytokines, MMP9, TIMPs, RANKL and OPG during orthodontic tooth movement. Eur J Orthod. 2013; 35(5):644-51.

48. Barbieri G, Solano P, Alarcón JA, Vernal R, Rios-Lugo J, Sanz M, Martín C. Biochemical markers of bone metabolism in gingival crevicular fluid during early orthodontic tooth movement. Angle Orthod. 2013; 83(1):63-9.

49. Capelli J Jr, Kantarci A, Haffajee A, Teles RP, Fidel R Jr, Figueredo CM. Matrix metalloproteinases and chemokines in the gingival crevicular fluid during orthodontic tooth movement. Eur J Orthod. 2011; 33(6):705-11.

50. Dudic A(1), Kiliaridis S, Mombelli A, Giannopoulou C. Composition changes in gingival crevicular fluid during orthodontic tooth movement: comparisons between tension and compression sides. Eur J Oral SCi. 2006: 114(5):416-22.

51. Sari E, Uçar C. Interleukin 1 beta levels around microscrew implants during orthodontic tooth movement. Angle Orthod. 2007: 77(6):1073-78

52. Hamamcı N, Acun Kaya F, Uysal E, Yokuş B. Identification of interleukin 2, 6 , and 8 levels around miniscrews during orthodontic tooth movement. Eur J Orthod. 2012; 34(3):357-61.

53. Alikhani M, Raptis M, Zoldan B, Sangsuwon C, Lee YB, Alyami B, Corpodian C, Barrera LM, Alansari S, Khoo E, Teixeira C. Effect of micro-osteoperforations on the rate of tooth movement. Am J Orthod Dentofacial Orthop. 2013: 144:639-48

54. Kaya FA, Hamamcı N, Uysal E, Yokuş B. Identification of tumor necrosis factor-a levels around miniscrews during canine distalization. Korean $\lrcorner$ Orthod. 2011; 41(1):36-41.

55. Kaya FA, Hamamci N, Basaran G, Dogru M, Yildirim TT. TNF-a, IL-1 $\beta$ and IL-8 levels in tooth early levelling movement orthodontic treatment. J Int Dent Med Res. 2010; 3(3):116-21.

56. Iwasaki LR, Gibson CS, Crouch LD, Marx DB, Pandey JP, Nickel JC. Speed of tooth movement is related to stress and IL-1 gene polymorphisms. Am J Orthod Dentofacial Orthop. 2006; 130:698.e1-e9.

57. Rody WJ, Wijegunasinghe M, Wiltshire WA, Dufault B. Differences in the gingival crevicular fluid composition between adults and adolescents undergoing orthodontic treatment. Angle Orthod. 2014; 84:120-26.

58. Gastel JV, Teughels W, Quirynen M, Struyf S, Damm V, Coucke W, Carels C. Longitudinal changes in gingival crevicular fluid after placement of fixed orthodontic appliances. Am J Orthod Dentofacial Orthop. 2011; 139:735-44. 
59. Tuncer BB, Ozdemir BC, Boynueğri D, Karakaya IB, Erguder I, Yucel AA, Aral LA, Ozmeric N. OPG-RANKL levels after continuous orthodontic force. GMJ. 2013; 24:33-6.

60. Lowney JJ, Norton LA, Shafer DM, Rossomando EF. Orthodontic forces increase tumor necrosis factor alpha in the human gingival sulcus. Am $J$ Orthod Dentofacial Orthop. 1995; 108(5):519-24.

61. Oshiro T, Shiotani A, Shibasaki Y, Sasaki T. Osteoclast induction in periodontal tissue during experimental movement of incisors in osteoprotegerin-deficient mice. Anat Rec. 2002; 266(4):218-25.

62. Kanzaki H, Chiba M, Arai K, Takahashi I, Haruyama N, Nishimura M, Mitani H. Local RANKL gene transfer to the periodontal tissue accelerates orthodontic tooth movement. Gene Ther. 2006; 13:678-85.

63. Kanzaki H, Chiba M, Shimizu Y, Mitani H. Periodontal ligament cells under mechanical stress induce osteoclastogenesis by receptor activator of nuclear factor kappaB ligand up-regulation via prostaglandin E2 synthesis. J Bone Miner Res. 2002; 17:210-20.

64. Nakao K, Goto T, Gunjigake KK, Konoo T, Kobayashi S, Yamaguchi K. Intermittent force induces high RANKL expression in human periodontal ligament cells. J Dent Res. 2007; 86:623-28.

65. Wise GE, King GJ. Mechanisms of tooth eruption and orthodontic tooth movement. J Dent Res. 2008; 87(5):414-34.

66. Kanzaki H, Chiba M, Shimizu Y, Mitani H. Dual regulation of osteoclast differentiation by periodontal ligament cells through RANKL stimulation and OPG inhibition. J Dent Res. 2001; 80(3):887-91.

67. Boyce BF, Xing L. Functions of RANKL/RANK/OPG in bone modeling and remodeling. Arch Biochem Biophys. 2008; 473:139-46.

68. Yano S, Mentaverri R, Kanuparthi D, Bandyopadhyay S, Rivera A, Brown EM, Chattopadhyay N. Functional expression of chemokine receptors in osteoblasts: role of regulated upon activation, normal T cell expressed and secreted (RANTES) in osteoblasts and regulation of its secretion by osteoblasts and osteoclasts. Endocrinology. 2005; 146:2324-35.

69. Yu X, Huang Y, Collin-Osdoby P. CCR1 chemokines promote the chemotactic recruitment, RANKL development, and motility of osteoclasts and are induced by inflammatory cytokines in osteoblasts. J Bone Miner Res. 2004; 19:2065-77.

70. Kindle $L$, Rothe $L$, Kriss $M$. Human microvascular endothelial cell activation by IL-1 and TNF-alpha stimulates the adhesion and transendothelial migration of circulating human CD14 monocytes that develop with RANKL into functional osteoclasts. J Bone Miner Res. 2006; 21:193-206.

71. Ren Y, Maltha JC, Kuijpers-Jagtman AM. Optimum force magnitude for orthodontic tooth movement: a systematic literature review. Angle Orthod. 2003; 73(1):86-92.

72. Maltha JC, Van Leeuwen EJ, Dijkman GE, Kuijpers-Jagtman AM. Incidence and severity of root resorption in orthodontically moved premolars in dogs. Orthod Craniofac Res. 2004; 7(2):115-21.

73. Von Böhl M, Maltha JC, Von Den Hoff JW, Kuijpers-Jagtman AM. Focal hyalinization during experimental tooth movement in beagle dogs. Am J Orthod Dentofacial Orthop. 2004; 125(5):615-23.

74. Teixeira CC, Khoo E, Tran J, Chartres I, Liu Y, Thant LM, Khabensky I, Gart LP, Cisneros G, Alikhani M. Cytokine expression and accelerated tooth movement. J Dent Res. 2010; 89(10):1135-41.

75. Ren Y, Vissink A. Cytokines in crevicular fluid and orthodontic tooth movement. Eur J Oral Sci. 2008; 116(2):89-97.

76. Celebia AA, Demirerb S, Catalbasc B, Arikand S. Effect of ovarian activity on orthodontic tooth movement and gingival crevicular fluid levels of interleukin-1 $\beta$ and prostaglandin E2 in cats. Angle Orthod. 2013; 83:70-5.

77. Monga N, Chaurasia S, Kharbanda OP, Duggal R, Moganty R. A study of interleukin $1 \beta$ levels in peri-miniscrew crevicular fluid (PMCF). Prog Orthod. 2014; 15(1):30-36.

78. Shetty N, Patil AK, Ganeshkar SV, Hegde S. Comparison of the effects of ibuprofen and acetaminophen on $\mathrm{PGE}_{2}$ levels in the GCF during orthodontic tooth movement: a human study. Prog Orthod. 2013; 14:6-10.

doi:10.1186/s40510-014-0065-6

Cite this article as: Kapoor et al:: Effect of orthodontic forces on cytokine and receptor levels in gingival crevicular fluid: a systematic review. Progress in Orthodontics 2014 15:65.

\section{Submit your manuscript to a SpringerOpen ${ }^{\odot}$ journal and benefit from:}

- Convenient online submission

- Rigorous peer review

- Immediate publication on acceptance

- Open access: articles freely available online

- High visibility within the field

- Retaining the copyright to your article

Submit your next manuscript at $>$ springeropen.com 